\title{
Facilitative Effects of the Ampakine CX516 on Short-Term Memory in Rats: Correlations with Hippocampal Neuronal Activity
}

\author{
Robert E. Hampson, ${ }^{1}$ Gary Rogers, ${ }^{2}$ Gary Lynch, ${ }^{3}$ and Sam A. Deadwyler ${ }^{1}$ \\ ${ }^{1}$ Department of Physiology and Pharmacology, Wake Forest University School of Medicine, Winston Salem, North \\ Carolina 27157, ${ }^{2}$ Cortex Pharmaceuticals, Irvine, California 92718, and ${ }^{3}$ Department of Psychiatry, University of \\ California, Irvine, California 92715
}

In the companion article (Hampson et al., 1998), the ampakine CX516 (Cortex Pharmaceuticals) was shown to produce a marked facilitation of performance of a spatial delayednonmatch-to-sample (DNMS) task in rats. Injections of the drug before each daily session produced a marked and progressive improvement in performance at longer delays ( $>5 \mathrm{sec}$ ) that persisted for $7 \mathrm{~d}$ after drug treatment was terminated. In most animals $(n=9)$ the increase in performance carried over to the intervening vehicle for days, whereas in others $(n=3)$ the effects dissipated within the session according to the pharmacological half-life of CX516. In this article we report firing correlates of simultaneously recorded cells in the CA1 and CA3 fields of the hippocampus over the period in which DNMS performance was facilitated by CX516. Sample and Delay period firing was enhanced by 100-350\% under CX516 and in- creased progressively over days as did DNMS performance. The firing increases were restricted to correct trials only and were largest on trials with long delays. Firing in the intertrial interval was also altered, but in a manner consistent with a previously demonstrated reduction in between-trial proactive interference by CX516. Finally, in animals in which the effects of CX516 were restricted to when the drug was actually present (i.e., no carryover effects), increased cell firing also paralleled the time course of the performance increase. Results are discussed with respect to the actions of ampakines on hippocampal cellular and synaptic processes that underlie DNMS performance.

Key words: ampakine; hippocampus; learning; memory; neuron ensembles; AMPA receptors
Extensive electrophysiological investigations in rodents and monkeys show that hippocampal neurons are "engaged" during "delay"-type memory tasks (Cahusac et al., 1989; Miller et al., 1991, 1996; Otto and Eichenbaum, 1992; Hampson et al., 1993; Rolls et al., 1993; Colombo and Gross, 1994). This suggests that hippocampal neurons may encode relevant aspects of tasks in which retention of specific information across an interposed delay interval is required. However, unlike in monkeys (Watanabe and Niki, 1985; Cahusac et al., 1993; Nishijo et al., 1993; Ono et al., 1993; Rolls et al., 1993; Colombo and Gross, 1994) and possibly humans (Fried et al., 1997), spatial attributes of stimulus situations in rodents appear to be encoded with a marked priority over nonspatial features by hippocampal neurons (cf. Muller, 1996), although nonspatial, task-relevant correlates have been reported in rodents during delay-type tasks (Otto et al., 1992; Sakurai, 1990; Deadwyler et al., 1996a). If hippocampal removal is necessary but not sufficient to show marked memory deficits (Squire, 1992; Zola-Morgan and Squire, 1993), then it follows that hippocampal neuronal activity must be only one link in many different circuits involved in neural representation of information during short-term memory tasks (Fuster, 1997).

Recent studies (Deadwyler et al., 1996a) have identified a

Received Oct. 17, 1997; revised Jan. 15, 1998; accepted Jan. 16, 1998.

This work was supported by National Institute on Drug Abuse Grants DA03502 and DA00119 to S.A.D. and DA08549 to R.E.H., and by Cortex Pharmaceuticals. We thank Douglas R. Byrd, Joanne K. Konstantopoulos, and Janet R. Brooks for technical assistance.

Correspondence should be addressed to Sam A. Deadwyler, Department of Physiology and Pharmacology, Wake Forest University School of Medicine, Medical Center Boulevard, Winston Salem, NC 27157-1083.

Copyright (ㄷ) 1998 Society for Neuroscience $\quad 0270-6474 / 98 / 182748-16 \$ 05.00 / 0$ major involvement of hippocampal neurons in the spatial delayednonmatch-to-sample (DNMS) task described in the companion article (Hampson et al., 1998). Ensemble-type analyses of multineuron recordings revealed that hippocampal cells encode features within the DNMS task consistent with dichotomized representation(s) of critical elements (i.e., sample vs nonmatch phase, left vs right lever position, etc.) necessary for effective performance (Deadwyler and Hampson, 1995). Error analyses using ensemble-derived firing components identified consistent relationships between strength of encoding of the Sample stimulus and performance on correct versus error trials (Hampson and Deadwyler, 1996a). It was determined therefore that hippocampal ensembles represent task relevant features via changes in spatiotemporal discharge patterns for specific events within that trial (Deadwyler et al., 1996a). The marked facilitation of DNMS task performance produced by the ampakine CX516 reported in the companion article (Hampson et al., 1998) affords a unique opportunity to assess directly whether changes in hippocampal neuronal activity are functionally associated with behavioral improvement (rather than impairment) in the same short-term memory task. If previous studies of hippocampal neuronal activity in this task are representative, then facilitation of performance by CX516 should be manifested by enhanced electrophysiological correlates of successful DNMS performance (Deadwyler et al., 1996a,b; Hampson and Deadwyler, 1996a; Deadwyler and Hampson, 1997).

\section{MATERIALS AND METHODS}

Detailed descriptions of the apparatus, behavioral training, and drug preparation have been included in the companion paper (Hampson et al., 1998). Briefly, male Long-Evans rats ( $n=18$; age, 200-250 d) were 
water-deprived but allowed free access to food, with a $20-22 \mathrm{hr}$ water deprivation regimen throughout the duration of DNMS training and recording. All animals were trained to the same criteria of $85 \%$ correct responses (on DNMS trials with delays of 1-5 sec) before and after surgery was performed (Hampson et al., 1998). The apparatus was a $43 \times$ $43 \times 53 \mathrm{~cm}$ Plexiglas behavioral testing chamber with retractable levers mounted on opposite halves of a wall, a water trough mounted between the levers, a nosepoke device on the opposite wall, a cue light positioned immediately above the nosepoke device, and house lights and "white noise" speaker mounted on top of the chamber. The apparatus was controlled by a minicomputer that collected behavioral and electrophysiological data onto computer disks.

Behavior. Animals were trained in the DNMS task as described in Hampson et al. (1998). DNMS trials were initiated by the extension of a single left or right lever, signaling the start of the Sample phase. After the Sample response (SR), the lever was retracted, and the Delay phase was initiated with the illumination of the cue light. The length of delay varied from 1 to $40 \mathrm{sec}$ on any trial, and the animal was required to nosepoke while the cue light was on to complete the delay. At the completion of the delay interval, the animal had to nosepoke once more [last nosepoke (LNP)] to extinguish the cue light and start the Recognition phase in which both levers were extended simultaneously. A response on the opposite lever to the SR, i.e., a Nonmatch response (NR), was rewarded, whereas a response on the same lever as the SR resulted in no reinforcement and a $5 \mathrm{sec}$ "time-out," during which chamber lighting was extinguished. A new trial was initiated after the $10 \mathrm{sec}$ intertrial interval (ITI) after a correct trial, or $5 \mathrm{sec}$ ITI after the timeout following an error trial.

Drug preparation and administration. Stock solutions of CX516 (35 $\mathrm{mg} / \mathrm{ml}$ ) were prepared in a $25 \% \mathrm{w} / \mathrm{v}$ solution of cyclodextrin vehicle (2-hydroxypropyl- $\beta$-cyclodextrin; Research Biochemicals, Natick, MA), and sonicated to ensure thorough mixing of the solution. Animals received $1 \mathrm{ml} / \mathrm{kg}$ intraperitoneal injections of the $35 \mathrm{mg} / \mathrm{ml} \mathrm{CX} 516$ stock solution (for a total of $35 \mathrm{mg} / \mathrm{kg}$ ) on drug administration days, or $1 \mathrm{ml} / \mathrm{kg}$ intraperitoneal injections of $25 \% \mathrm{w} / \mathrm{v}$ cyclodextrin solution on vehicleonly days. All injections were given $\sim 5 \mathrm{~min}$ before the start of the behavioral session. Vehicle solutions were prepared every $5 \mathrm{~d}$, and CX516 solutions were prepared fresh on the day of administration.

Surgery. Each animal was anesthetized with ketamine $(100 \mathrm{mg} / \mathrm{kg})$ and xylazine $(10 \mathrm{mg} / \mathrm{kg})$ and stereotaxically implanted with a specially designed multiple microwire $(50 \mu \mathrm{m})$ electrode array (NBLabs, Denison, TX) (Deadwyler et al., 1996a). The array was positioned at the time of surgery with the tips of the electrodes above or within the cell layers of the CA1 and CA3 subfields of the hippocampus. The center pair of array electrodes was positioned at coordinates $3.8 \mathrm{~mm}$ posterior to bregma, 3.0 $\mathrm{mm}$ left of midline. The longitudinal axis of the area was angled $30^{\circ}$ from midline, with posterior electrode sites more lateral than anterior sites. The array was driven in $25 \mu \mathrm{m}$ steps to a depth of $3.0-4.0 \mathrm{~mm}$ for CA3 leads, with the CA1 leads automatically positioned $1.2-1.4 \mathrm{~mm}$ higher. Neural activity from the microwire electrodes was monitored throughout surgery to ensure placement near the hippocampal cell layers (Heyser et al., 1993; Deadwyler et al., 1996a). After array placement, the cranium was sealed with bone wax and dental cement, and the animal was allowed to recover. The scalp wound was treated periodically with Neosporin antibiotic, and animals were given an injection of Crysticillin (penicillin G, $300,000 \mathrm{U})$ to prevent infection. All animal care and experimental procedures conformed to National Institutes of Health and Society for Neuroscience guidelines for care and use of experimental animals.

Multi-neuron recording technique. Neural activity (extracellular action potentials, or "spikes") and behavioral responses were digitized and time-stamped for computer processing in relation to successive behavioral "events" within each DNMS trial. Six to nine neurons, one from each wire, were isolated and selected for analysis from the 16 different locations on the recording array (Hampson et al., 1992, 1993, 1994). Neuronal action potentials were digitized at $40 \mathrm{kHz}$ and isolated by time-amplitude window discrimination as well as computer-identified individual waveform characteristics via a Spectrum Scientific Spike Sorter (Plexon, Dallas, TX). Identified spikes from selected wires were "tracked" from session to session by waveform and firing characteristics within the task (perievent histograms). It is possible that the neuronal spikes discriminated on a given wire may not have consistently identified the same neuron (McNaughton et al., 1983). However, only spike waveforms with associated firing rates and perievent histograms (i.e., behavioral correlates) that were consistent across sessions were included in the analysis. The likelihood that the same 10 neurons were not continuously recorded under these conditions was considered extremely low (Hampson and Deadwyler, 1996a).

Analysis. Changes in neural firing rates were analyzed for statistically significant differences via two- and three-way ANOVA. Measurements of single neuron firing rate included mean $( \pm$ SEM) firing rate within defined intervals (i.e., across delays in 5-10 sec blocks, or across the ITI), mean firing rate before, during, and after fixed behavioral events (i.e., $\pm 1.5 \mathrm{sec}$ around SR or NR), and peak firing rate at fixed behavioral events (i.e., SR or NR). Firing rates were analyzed by multivariate firing tendencies. Individual mean comparisons used pairwise linear contrasts within the ANOVA.

\section{RESULTS \\ Effects of CX516 on hippocampal cell firing during DNMS performance}

The following questions were the most critically relevant to the effects of CX516 on hippocampal activity during DNMS performance: (1) was the improvement in behavioral performance associated with administration of CX516 always accompanied by a change in task-relevant firing of hippocampal neurons, and conversely (2) were there circumstances in which the effects of CX516 on DNMS performance could be dissociated from the effects on hippocampal cellular activity?

The effects of CX516 on hippocampal firing were assessed initially via standard two- and three-way ANOVA tests of changes in firing rate of 56 individual neurons recorded in the "carryover" group of the nine different animals discussed in the companion article (Hampson et al., 1998). There was a highly significant main effect $\left(F_{(15,800)}=101.47 ; p<0.001\right)$ of CX516 treatment on overall mean firing rate within the trial (across all treatments, animals, and sessions) that was accompanied by significant, simple effect influences of drug administration (treated vs untreated animals: $\left.F_{(2,800)}=526.19 ; p<0.001\right)$, stage of testing (i.e., Pre-, during, or Post-CX516 administration: $F_{(7,800)}=112.27 ; p<$ $0.001)$, and significant session $\times$ drug interaction $\left(F_{(22,800)}=30.0\right.$; $p<0.001)$. There were no significant differences in mean firing rate across animals in the Pre-CX516 condition $\left(F_{(47,800)}=1.53\right.$; NS), nor were there significant animal $\times$ drug or animal $\times$ session interactions $\left(F_{(47,800)} \leq 1.21\right.$; NS). Specific comparisons showed highly significant differences in overall firing within a trial between the CX516 versus the vehicle Control group $\left(F_{(1,800)}=\right.$ $169.31 ; p<0.001)$ and between Pre-CX516 versus Post-CX516 in the CX516 group $\left(F_{(1,800)}=122.11 ; p<0.001\right)$ but not between the Control and Pre-CX516 condition $\left(F_{(1,800)}=181.96 ; p<\right.$ 0.001).

As reported previously (Deadwyler et al., 1996b, 1997), the major increases in firing were during the Sample (Pre-CX516 = $2.73 \pm 0.21 \mathrm{~Hz}$; Post-CX516 = 8.81 $\pm 0.43 \mathrm{~Hz} ; F_{(1,800)}=217.0$; $p<0.001$ ), Delay (Pre-CX516 = $1.75 \pm 0.27 \mathrm{~Hz}$; Post-CX516 = $\left.5.45 \pm 0.42 \mathrm{~Hz} ; F_{(1,800)}=80.61 ; p<0.001\right)$, and Recognition $($ Pre-CX516 = $4.07 \pm 0.41 \mathrm{~Hz}$; Post-CX516 = $11.41 \pm 0.61 \mathrm{~Hz}$; $\left.F_{(1,800)}=317.28 ; p<0.001\right)$ phases of the DNMS trial. Background firing rate, assessed 5-15 sec after the Sample response (SR) and in the post-Recognition phase, was not significantly different (see below).

\section{Changes in task-specific firing across days of CX516 administration}

In the companion article (Hampson et al., 1998), behavioral performance was enhanced across the time period of CX516 administration (days 8-25; Fig. 1A). Within-trial firing characteristics were assessed by plotting the mean change in firing across all neurons during different phases of the task on days when CX516 was administered and on intervening vehicle days. Figure 
A.

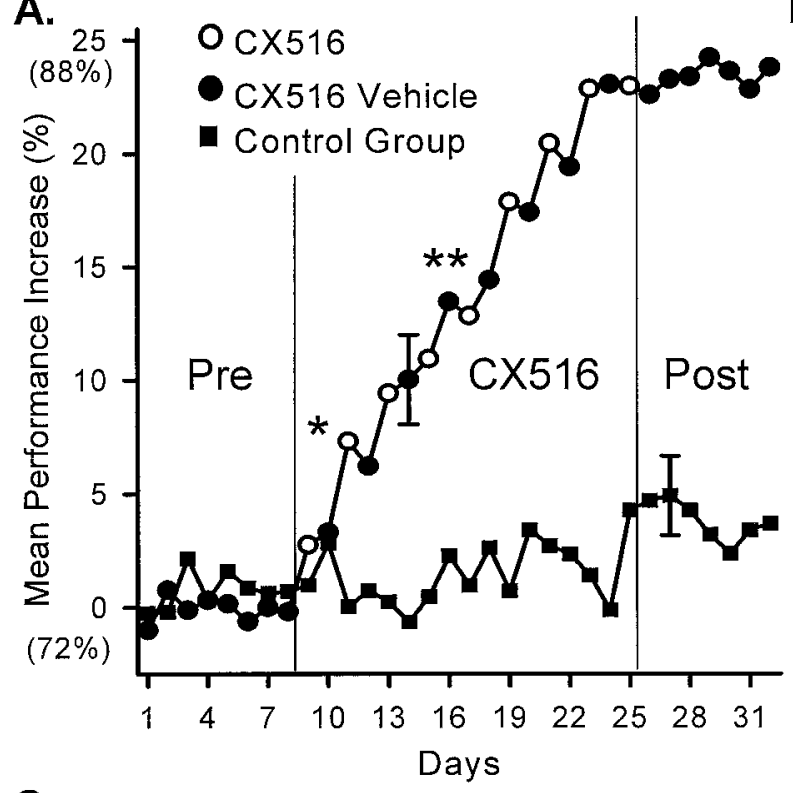

C.

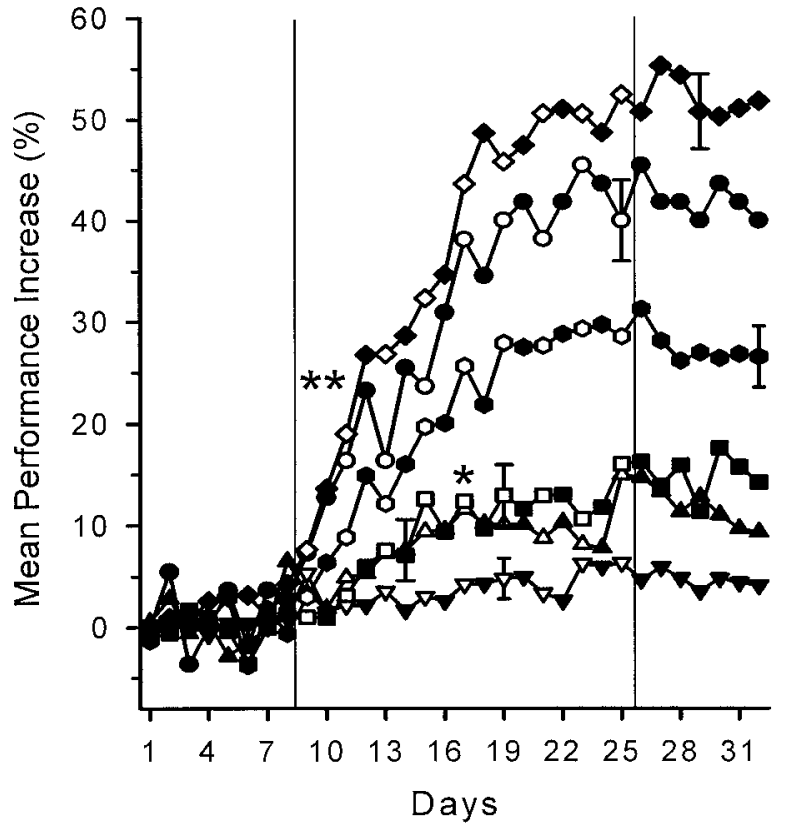

B.

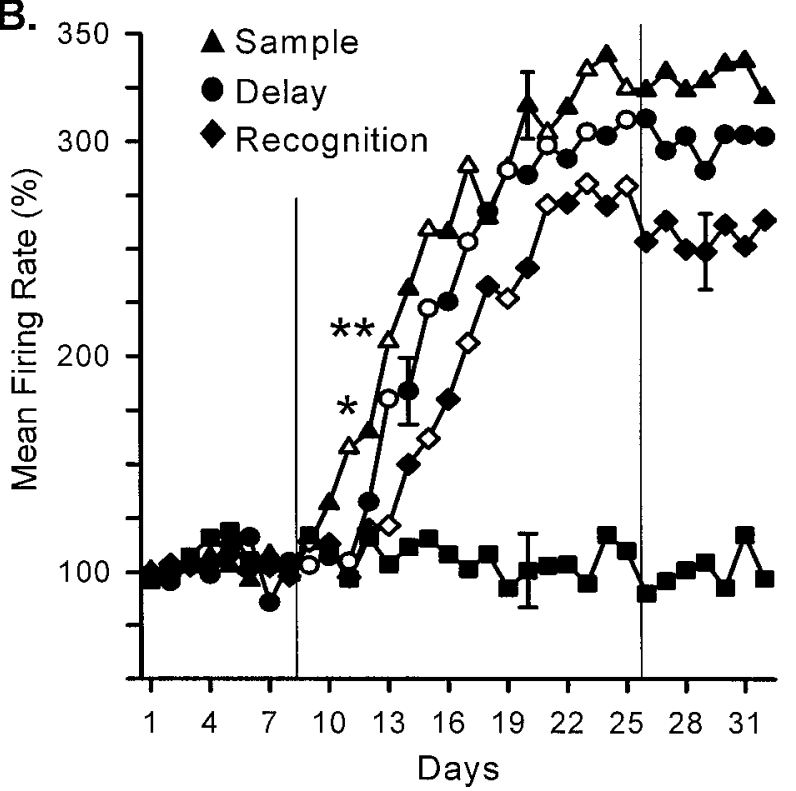

D.

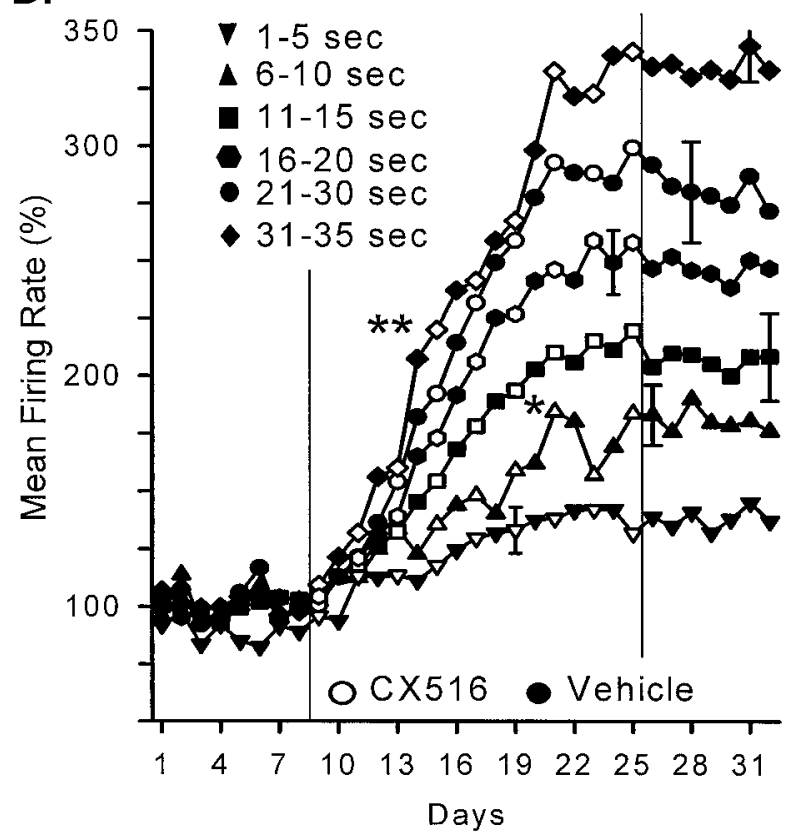

Figure 1. Time course of change in DNMS performance and concomitant hippocampal cell firing during CX516 testing. $A$, Mean DNMS performance over the entire $32 \mathrm{~d}$ testing period (Pre, CX516, and Post) (Hampson et al., 1998) for the CX516 carryover ( $n=9$; open circles, drug days; filled circles, vehicle-only days) and vehicle control $(n=6$; filled squares) groups. Error bars indicate largest SEM for each group. Vertical lines demarcate CX516 treatment period. Asterisks indicate individual sessions with significant increase in performance from Control group. ${ }^{*} p<0.01 ; * * p<0.001 . B$, Change in hippocampal cell firing rate in Sample (triangles), Delay (circles), and Recognition (diamonds) phases of the DNMS task plotted over the $32 \mathrm{~d}$ testing period. Means calculated for 56 hippocampal neurons (6 per animal) in CX516 group. Filled squares show mean firing rate in the Delay phase of the DNMS task for 24 other neurons recorded from six animals in the Control group. All firing rates are plotted as percentage of predrug baseline firing rate $(100 \%)$ across animals. Symbols and vertical lines same as in A. Asterisks indicate individual sessions with significant firing rate increases over Control group. ${ }^{*} p<0.01 ;{ }^{*} p<0.001$. $C$, Performance data for the same nine animals in the CX516 group as in $A$ sorted according to performance at different delays [same as Fig. 4 in companion article (Hampson et al., 1998)]. Performance is plotted as percent increase over predrug baseline for each delay in CX516 group only. Asterisks indicate significant increases in individual session performance from Control. ${ }^{*} p<0.01 ; * *<<0.001 . D$, Mean change in firing rate for the neurons shown in $B$ plotted for the same delay intervals as shown in $C$ as percentage of Pre-CX516 firing. Open symbols indicate drug days; filled symbols indicate vehicle days. All symbols represent the average of mean rate for all 56 neurons. Error bars represent largest SEMs per curve. Asterisks indicate significant increases in firing from Control group. ${ }^{*} p<0.01 ;{ }^{* *} p<0.001$.

$1 B$ shows the associated changes in mean firing rates in the three main phases of the DNMS trial (Sample, Delay, and Recognition) for the 56 neurons recorded from the same nine animals shown in Figure $1 \mathrm{~A}$. The separate curves reflect a $222 \%$ mean increase in
Sample (SP-SR interval; Fig. 2) phase firing $\left(F_{(1,800)}=53.64 ; p<\right.$ $0.001)$, a $205 \%$ mean increase $\left(F_{(1,800)}=45.13 ; p<0.001\right)$ in Delay (SR-LNP) interval firing, and a $180 \%$ mean increase $\left(F_{(1,800)}=35.18 ; p<0.001\right)$ in firing in the Recognition (LNP- 
NR) phase across all test sessions (days 9-25). In contrast, the lack of an increase in DNMS performance in the Control group (Fig. $1 A$ ) was mirrored by the absence of a significant change in firing rate $\left(n=6\right.$ animals; 24 neurons; $\left.F_{(1,800)}=0.71 ; \mathrm{NS}\right)$ at any time over the entire testing period.

As shown in Figure $1 B$, the CX516-related firing increase in each phase of the task was not the same, as indicated by the different time course of changes across testing days for each phase of the DNMS task. Significant changes in firing rate from PreCX516 and the Control group means appeared first in the Sample phase on day 11 (Fig. $1 B$, asterisk) ( $>75 \%$ increase; $p<0.01$ ), followed by increased Delay interval firing (day 13) and finally by elevations in the Recognition phase (day 15). It is important to note that the order of these firing increases did not differ across animals, indicating that the phase-related sequence of changes represented a similar process in each animal. Figure $1 B$ also shows sustained increased firing as a physiological correlate of the behavioral "carryover" effect on intervening vehicle days during CX516 administration described in the companion article (Hampson et al., 1998). The "carryover" effect was manifested in hippocampal cell firing in all three phases of the task. In addition, firing was maintained in all three phases on the 7 Post-CX516 days after drug termination (days 26-32) at the same firing rate (Sample $>$ Delay $>$ Recognition), as seen on the final days of CX516 administration (Fig. 1B).

In the companion article (Hampson et al., 1998), it was also noted that an important feature of the influence of ampakines on performance was the differential improvement with respect to length of delay on a given trial. DNMS performance was enhanced more on trials with longer $(16-35 \mathrm{sec})$ versus shorter (6-15 sec) delays over the time course of CX516 administration and afterward as shown in Figure $1 C$. Figure $1 D$ shows the corresponding effect of Delay on firing rate for the same trials as shown in Figure $1 C$. The rank order of firing increase across the Delay categories was the same as for behavioral performance during the administration of CX516. As with DNMS performance, these increases in Delay firing persisted after CX516 treatment was terminated. Comparison of Pre- versus PostCX516 firing on trials with delay intervals of 1-5 sec showed no significant differences in accordance with the behavioral data $($ Pre-CX516 = $1.23 \pm 0.16 \mathrm{~Hz}$; Post-CX516 = $1.72 \pm 0.13 \mathrm{~Hz}$; $F_{(1,800)}=2.09 ;$ NS). Trials with delay intervals of 6-10 sec showed slightly larger differences (Pre-CX516 = $1.29 \pm 0.14 \mathrm{~Hz}$; PostCX516 $\left.=2.13 \pm 0.15 \mathrm{~Hz} ; F_{(1,800)}=6.25 ; p<0.02\right)$, compared with marginal changes in performance (Fig. $1 C$ ). However, on trials with delay intervals $>10 \mathrm{sec}$, firing was significantly increased by CX516, including the $11-15 \mathrm{sec}$ (Pre-CX516 = $1.22 \pm$ $0.11 \mathrm{~Hz}$; Post-CX516 $\left.=2.45 \pm 0.14 \mathrm{~Hz} ; F_{(1,800)}=7.52 ; p<0.01\right)$, $16-20 \mathrm{sec}($ Pre-CX516 = $1.42 \pm 0.16 \mathrm{~Hz}$; Post-CX516 = $3.51 \pm$ $\left.0.13 \mathrm{~Hz} ; F_{(1,800)}=21.27 ; p<0.01\right), 21-30 \mathrm{sec}($ Pre-CX516 = $1.52 \pm 0.13 \mathrm{~Hz}$; Post-CX516 = $4.46 \pm 0.25 \mathrm{~Hz} ; F_{(1,800)}=50.90$; $p<0.001$ ), and 31-35 sec (Pre-CX516=1.95 $\pm 0.23 \mathrm{~Hz}$; Post-CX516 $\left.=6.41 \pm 0.27 \mathrm{~Hz} ; F_{(1,800)}=117.14 ; p<0.001\right)$ Delay intervals. Firing on trials with delays of $36-40 \mathrm{sec}$ did not differ from 31-35 sec (i.e., Post-CX516, 36-40 sec; mean rate $=6.79 \pm$ $0.30 \mathrm{~Hz} ; F_{(1,800)}=2.17$; NS), which corresponded to the fact that behavior performance on trials with 36-40 sec delays did not differ significantly from Pre-CX516 levels (Hampson et al., 1998). Firing on trials with delays $<16$ sec was not significantly elevated until after day 20, as compared with day 13 for longer delays $(>16$ sec), suggesting that the same process did not underlie the firing increase at longer versus shorter delay intervals.
Figure 2 shows firing in six different simultaneously recorded neurons from a single animal in the carryover group. Averaged trial-based histograms (TBHs) are shown for only 30-40 sec delay trials, before and after CX516 administration. Superimposed individual waveforms of the six simultaneously recorded neurons are shown at the right of each respective TBH. The two sessions illustrated were recorded on day 8 Pre-CX516 and day 25 CX516, a span of $18 \mathrm{~d}$ over which trial-specific firing increased in each neuron. It is clear that the differential firing tendencies, especially in the Sample and Recognition phases of the task and the later portions of the Delay $(20-30 \mathrm{sec})$, were markedly increased after CX516 administration (CX516 in Fig. 2). Neurons 2 and 3 showed modest firing increases during appropriate taskrelevant events (SR, Delay, NR) in the Pre-CX516 period that were substantially elevated after CX516 administration. Other neurons in the same ensemble showed only marginal differential firing at very low rates in the Pre-CX516 period but were drastically altered by CX516 administration (Fig. 2, CX516, neurons 4, 5 , and 6 ). Figure 2 illustrates the fact that (1) background firing rate was slightly elevated across all recorded cells after CX516 exposure; (2) cells with only marginal firing tendencies before CX516 were substantially altered in task-relevant discharge characteristics; (3) robust firing cells before CX516 also showed increases, but the change was less dramatic; and (4) the pattern of firing across neurons became much more "coherent" during the DNMS trial after CX516 exposure. The consistency in recorded waveforms makes it likely that the same neurons were recorded over the $18 \mathrm{~d}$ testing period.

The composite TBHs summed over all six neurons, at the bottom of Figure 2, show that CX516 altered quantitatively the nature of ensemble firing but preserved the same qualitative features as before drug treatment. Figure 3 shows the development of firing rate increases in the TBHs of three simultaneously recorded neurons in a different animal over days 9-15 of CX516 administration and illustrates that elevated firing in the same neurons "carried over" to intervening vehicle days.

\section{CX516-induced changes from simple to complex firing patterns}

Encoding of left or right lever position in the TBHs was determined to be represented within neurons by distinctly different temporal discharge patterns in the SR. Figure 4 shows the distinction between Left and Right trials with respect to firing of a single neuron recorded before (Pre) and after CX516 administration. It is clear that before CX516 the cell fired only during the SR (Fig. 4; Left TBH), with differential peak latencies (asterisks) for left and right SRs, respectively, and no increased firing in either the Delay or the Recognition phases of the task on either trial type. This type of firing pattern has been defined as "singleevent," in that it distinguishes only one event within the trial (i.e., left lever position) from its complement (right lever position) on the other type of trial. After CX516, discharge increased in the other two phases, including Sample, with peak latency differences for different lever positions maintained. Robust firing also occurred in the Delay and Recognition phases, with position differentiated by firing intensity (NR amplitude) rather than latency. The emergence of firing at multiple phases of the task, with maintained differentiation with respect to position and other task characteristics, illustrates the transition from single- to "multievent" firing in the same neuron after exposure to CX516. The change in proportion of single-event to multi-event firing across all neurons was an increase from $29 \%$ in Pre-CX516 sessions to 

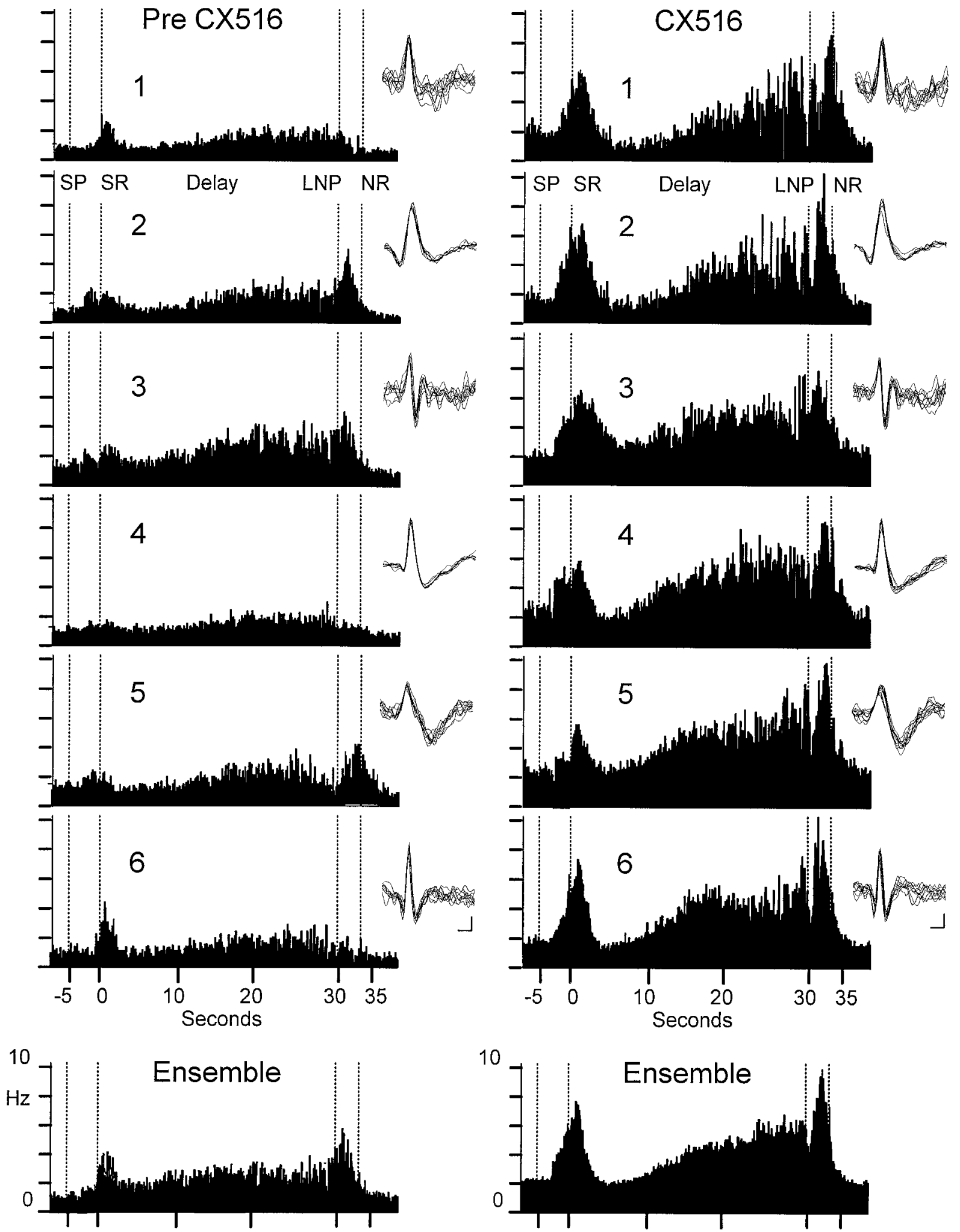

Figure 2. Change in trial-based histograms (TBHs) for a selected ensemble of six hippocampal CA3 and CA1 neurons recorded simultaneously in a single (carryover) animal before and after administration of CX516. Left, The top six traces represent TBHs for individual neurons recorded simultaneously from an ensemble. TBHs were constructed from 30-40 sec DNMS trials recorded on day 8 (Pre CX516). Activity was summed across all trials (i.e., one session), grouped in $100 \mathrm{msec}$ bins, and plotted continuously in time within the DNMS trial as mean spikes/second. The composite firing for the entire ensemble is shown in the bottom TBH (Ensemble) by averaging of the above TBHs from the six individual neurons. Each TBH is constructed from the presentation of the Sample lever $(S P) \sim 5 \mathrm{sec}$ before the Sample Response $(S R)$ through the interposed Delay interval if the trial was $30 \mathrm{sec}$, up to the Last Nosepoke (LNP) and nonmatch response (NR), terminating at the intertrial interval (ITI) 5 sec later. To accommodate trials with varying durations of the interposed delay, the 30-40 sec segments were not plotted. Each bin rate during the delay was normalized according to the number of averaged trials. Right, TBHs for the same neurons (1-6) averaged over the same number of 30 sec delay trials on (Figure legend continues) 
Neuron 1
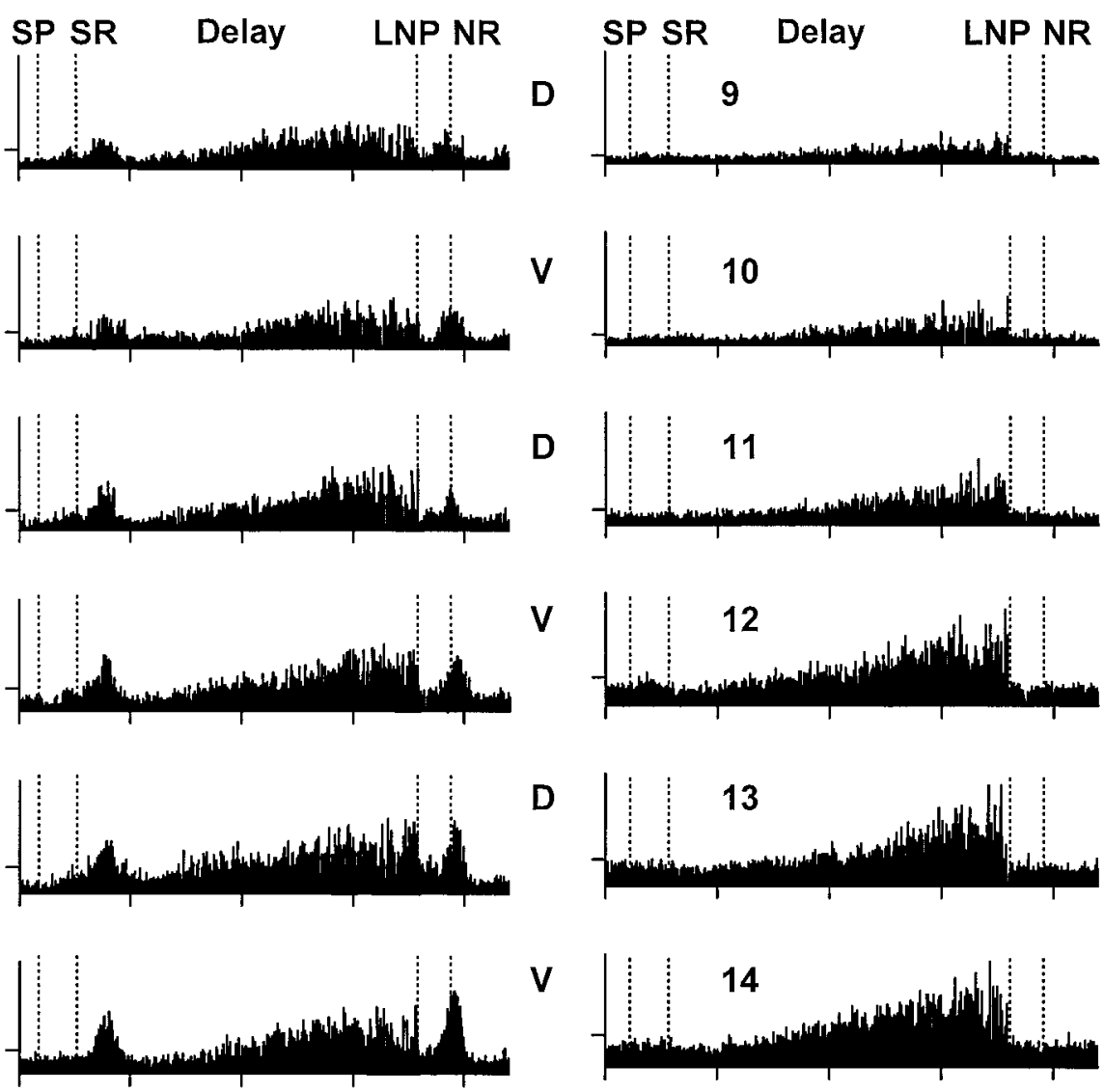

10
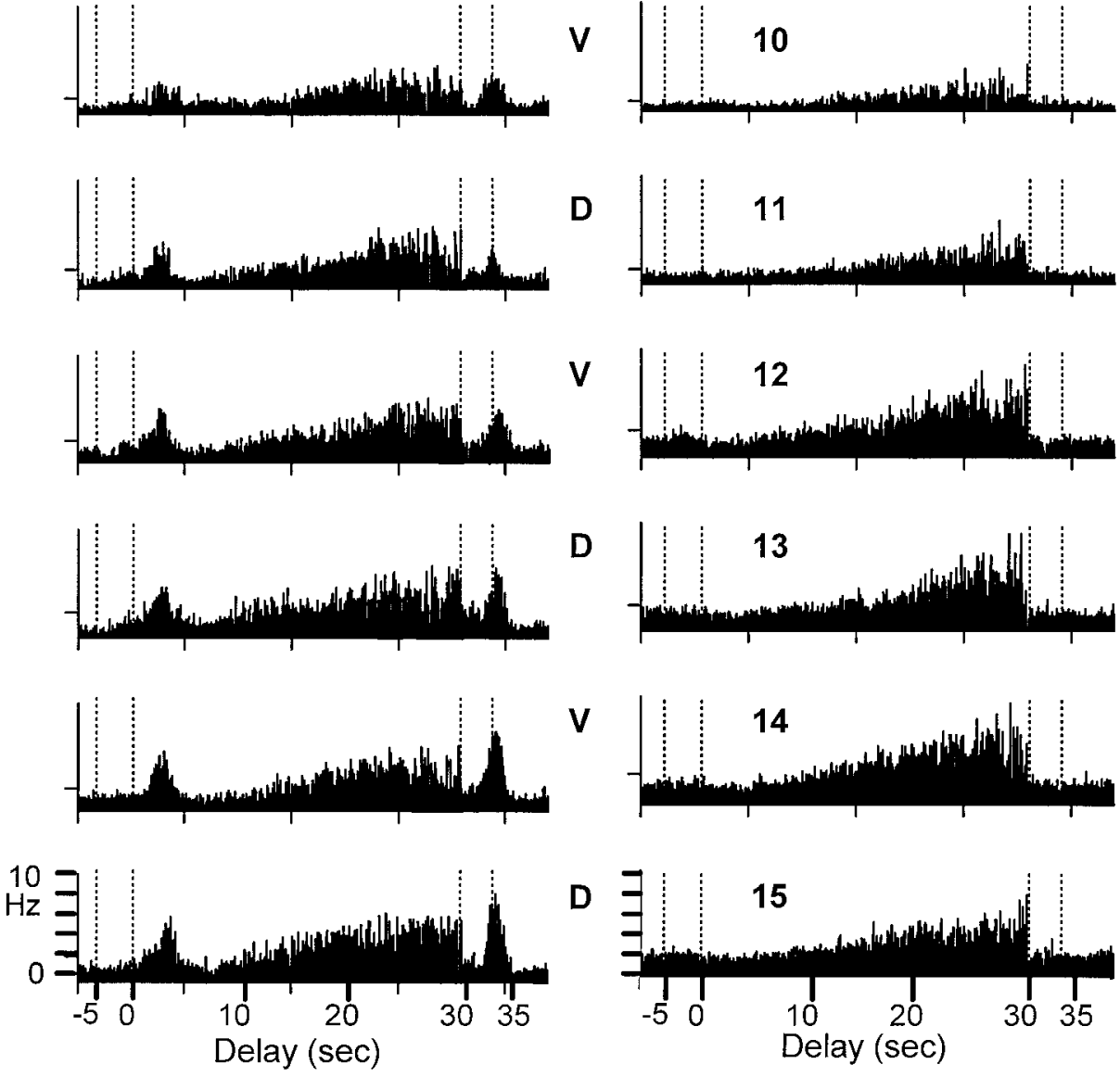
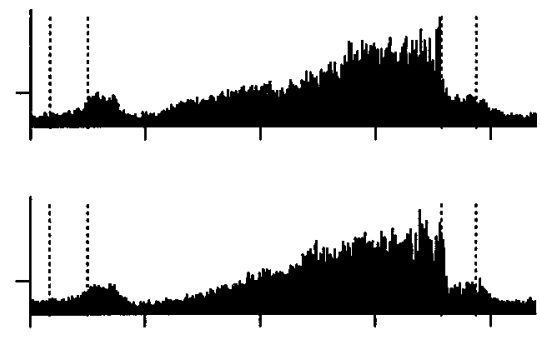

Neuron 3
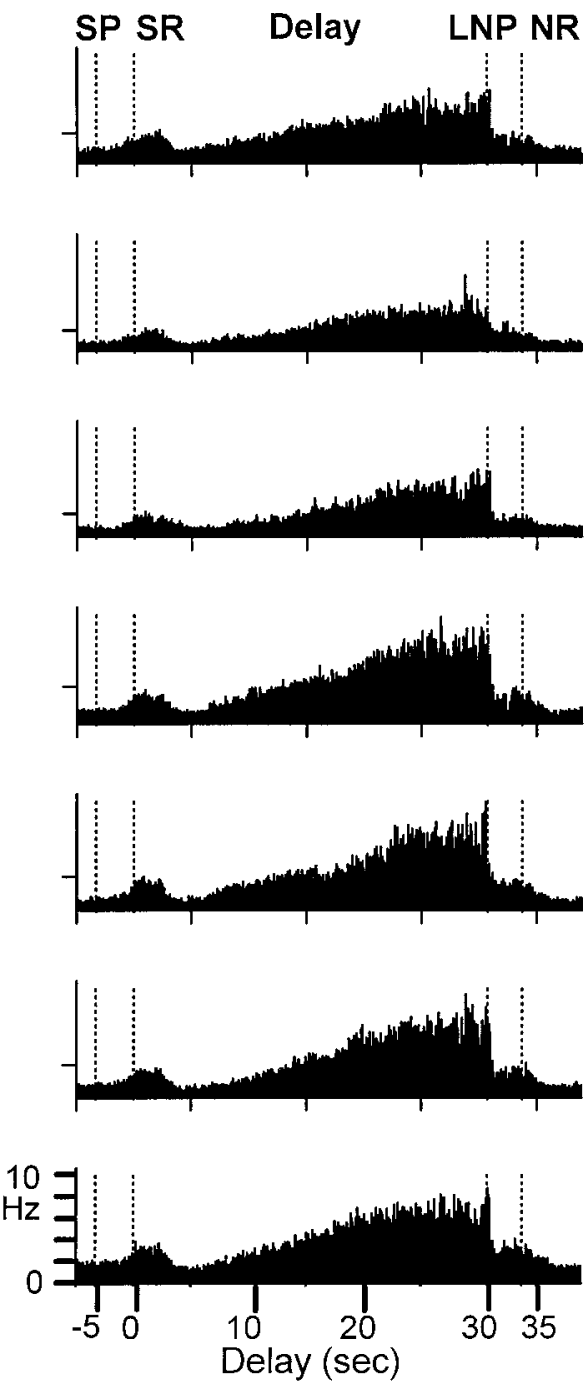

Figure 3. TBHs for three hippocampal neurons recorded from a different animal in the carryover group, plotted for $7 \mathrm{~d}$ of CX516 administration. TBHs were constructed for each neuron from sessions of 100 DNMS trials, all at delays of 30-40 sec (see Fig. 2) over the consecutive days numbered in the center column under Neuron 2 (i.e., $9=$ day $9,10=$ day 10 , etc.). Drug $(D)$ or vehicle $(V)$ days are indicated to the left of the middle TBHs. The same neurons were assumed to be recorded within each ensemble on consecutive days throughout the course of CX516 administration because there was no change in waveform or shape of TBH. Note persistent firing changes on vehicle days $(V)$ when CX516 was not administered. TBH construction and labels are the same as Figure 2.

$78 \%$ multi-event firing $\left(\chi^{2}=14.7 ; p<0.01\right)$ after CX516 administration.

\section{Noncarryover and hippocampal activity}

In the companion article (Hampson et al., 1998), it was shown that for unknown reasons, 3 of the 12 animals that received the same injection regimen of CX516 showed greatly reduced or minimal "carryover" of facilitated DNMS performance to intervening vehicle days. These three animals exhibited significant improvement in DNMS performance, but only during the first half (i.e.,
35-40 min) of each CX516 testing session. Performance in the second half was reduced to near predrug levels (Hampson et al., 1998; their Fig. 7). A critical test of the relationship of hippocampal activity to the behavioral effects of CX516 was therefore whether the transient improvement in the three noncarryover animals was also accompanied by biphasic changes in hippocampal cell firing on the same trials.

A three-way ANOVA showed that the overall within-trial firing rate in the first half of the CX516 session in these three "noncarryover" animals (Hampson et al., 1998) was significantly ele-

day 25 at the end of CX516 administration. Inset waveforms show the discriminated extracellular action potentials for each single neuron isolated from separate wires on the electrode array recorded on days 8 and 25, respectively. Calibration: $50 \mu \mathrm{V}, 200 \mu$ sec. Trial events are labeled in TBH of neuron 1 by dashed vertical lines. Horizontal axis of TBHs depicts time (bin width $=100 \mathrm{msec}$ ); vertical axis indicates mean firing rate in $\mathrm{Hz}$ normalized to the number of trials. 

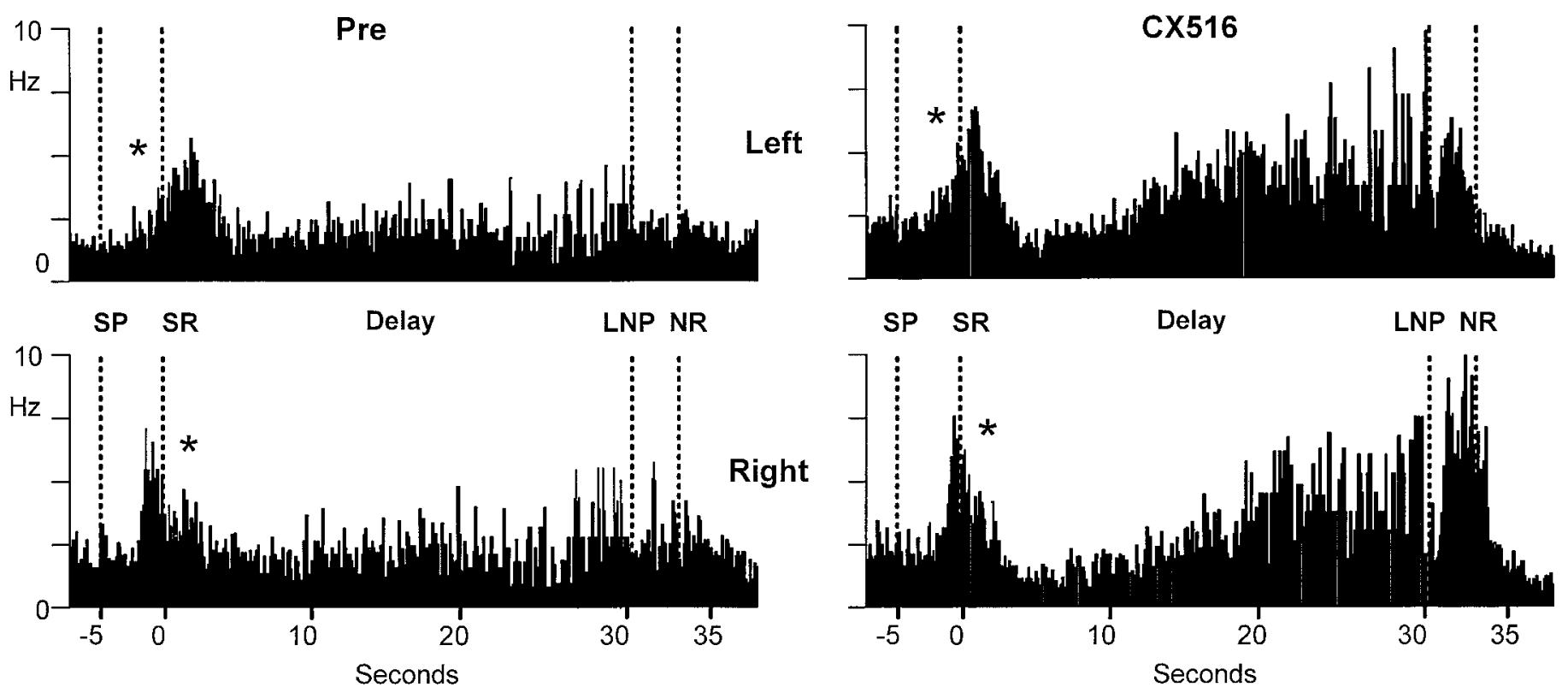

Figure 4. Transition from single- to multi-event firing pattern of a single hippocampal neuron before and after treatment with CX516. Pre, Single neuron TBHs recorded from an animal in the carryover group constructed (as in Fig. 2) from DNMS trials, but sorted according to firing on Left (top) or Right (bottom) Sample trials. Each TBH consisted of 50 DNMS trials of a given type recorded on day 8 of testing (Pre-CX516). Note increased firing restricted to the Sample phase (i.e., at the SR) of the trial, with no increase in firing during the Delay or Recognition phases. The asterisks on the Left or Right SRs indicate the differentiated peak discharge latency for those respective trial types. CX516, TBHs for the same neuron on Left versus Right trial types recorded on day 25 of testing (Post-CX516). Firing was expanded to include additional events within the DNMS task. Asterisks indicate that the same differential temporal discharge at the Sample response $(S R)$ was maintained on Left versus Right Sample trials. In addition, firing rate increased across the delay interval on both types of trial, and Left versus Right trials were further distinguished by differential amplitude of the NR firing in the same neuron. TBHs and labels were constructed as described above in Figure 2.

vated relative to the second half of the session (mean first half $=$ $5.22 \pm 0.67 \mathrm{~Hz}$; mean second half $=3.39 \pm 0.79 \mathrm{~Hz} ; F_{(1,431)}=$ $10.79 ; p<0.01)$ and to the Pre-CX516 baseline firing rate (mean baseline $\left.=2.31 \mathrm{~Hz} \pm 0.53 ; F_{(1,431)}=17.44 ; p<0.001\right)$. Also the overall firing rate in the first half of the drug session was significantly higher than during the first half of the intervening vehicle sessions (mean first half vehicle $=2.24 \pm 0.47 \mathrm{~Hz} ; F_{(1,431)}=$ $15.11 ; p<0.001)$. Firing in the first half-session for noncarryover animals also increased on successive drug days and was not significantly different from the carryover group across the first $5 \mathrm{~d}$ of CX516 exposure $\left(F_{(9,431)}=1.48\right.$; NS). However, on the last 4 drug days, firing in the noncarryover group was slightly lower than in the carryover group (mean noncarryover $=4.79 \pm 0.41 \mathrm{~Hz}$; mean carryover $\left.=5.88 \pm 0.52 \mathrm{~Hz} ; F_{(7,431)}=2.66 ; p<0.05\right)$.

The above daily firing changes closely matched the behavioral profile of the noncarryover group over the same testing days (Hampson et al., 1998, their Fig. 7). Firing rate in the second half of the session was marginally elevated relative to Pre-CX516 session rates (mean Pre-CX516 second half $=2.27 \pm 0.41 \mathrm{~Hz}$; $\left.F_{(1,431)}=3.97 ; p<0.05\right)$, but not when compared with the second half of intervening vehicle sessions (mean second half vehicle $=$ $\left.2.41 \pm 0.39 \mathrm{~Hz} ; F_{(1,431)}=2.19 ; \mathrm{NS}\right)$. The slightly increased firing in the second half of the drug session across days was consistent in all three animals $\left(F_{(4,431)}=1.72 ; \mathrm{NS}\right)$, and most likely reflected residual effects of CX516, the half-life of which was estimated at $40 \mathrm{~min}$ in a session of $90 \mathrm{~min}$ duration (Hampson et al., 1998, their Fig. 7).

Neurons recorded from the three animals in the noncarryover group exhibited the same firing correlates as animals in the carryover group before CX516 exposure (Fig. 2). Figure 5 shows six different neurons in an ensemble recorded from a noncar- ryover animal, in which most exhibited "single-event"-type firing. However, in contrast to animals in the carryover group (see above), exposure to CX516 did not change the neurons to a multi-event firing mode. Although neurons 1, 3, 5, and 6 in Figure 5 showed marked increases in firing rate (especially within the Delay), they did not extend their firing correlates to other task phases. Neurons 2 and 4 on the other hand, showed increased firing only in the single events that they encoded, and no change in rate in other portions of the trial during CX516 exposure. Of the total population of neurons $(n=20)$ in the noncarryover group, only $25 \%(n=5)$ showed multi-event firing correlates after exposure to $\mathrm{CX} 516$, which was very close to the percentage in the Pre-CX516 condition for the carryover group and overall for the control group. It is interesting that the composite ensemble TBHs before and after CX516 treatment for the Noncarryover group (Fig. 5, bottom) were similar to the composite TBHs for the Carryover group (Figs. 2, 5, compare Ensemble TBHs), indicating that the information represented across neurons in each group of animals was nearly the same. Therefore, much of the information represented by the larger percentage of multievent neurons in the carryover group was apparently "redundant" (Hampson and Deadwyler, 1996b; Deadwyler and Hampson, 1997), a circumstance that could have led to improved DNMS performance on intervening vehicle days.

To illustrate this transient facilitation of hippocampal ensemble firing in noncarryover animals, Figure 6 shows the first versus second half session changes in ensemble TBHs $(n=8$ neurons in the ensemble) from another animal in this group. During the first half of the drug session, firing was increased in the ensemble in the same manner as for multi-event neurons in the carryover group (Figs. 2, 3). However, in the second half of 

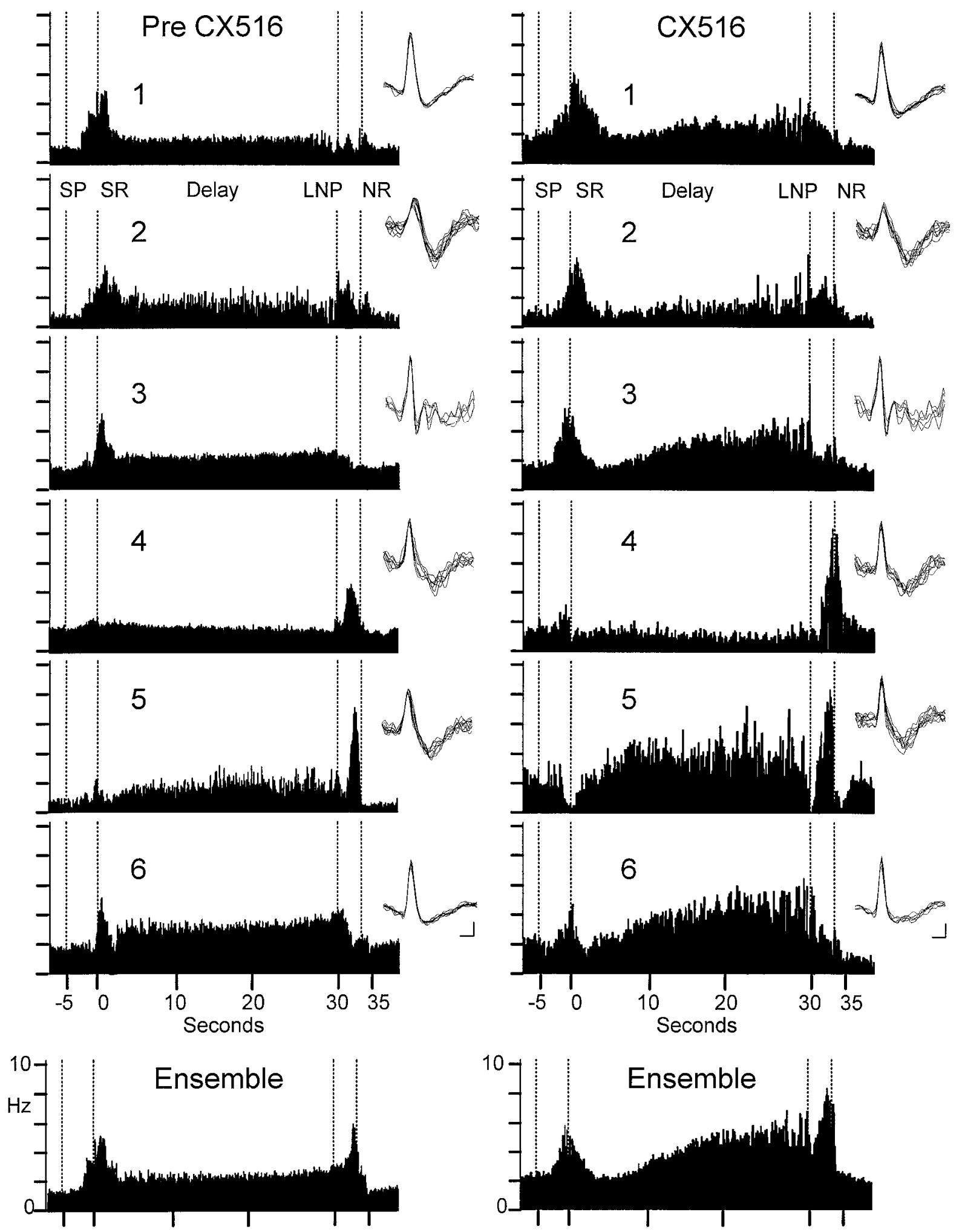

Figure 5. Individual TBHs from six hippocampal neurons recorded from an animal in the noncarryover group before and during exposure to CX516. Single neuron TBHs (top) were constructed as in Figure 2, with the Ensemble composite TBHs (bottom) reflecting the average of the six individual TBHs. Left, TBHs were constructed for each neuron during the first half of the session (50 trials) on Pre-CX516 day 8 . Note that each neuron exhibited "single-event" firing only with increased firing in the Sample or Recognition phase, but not both. However, the composite ensemble TBH (bottom) for neurons 1-6 demonstrated a firing pattern that included increased Sample, Delay, and Recognition phase firing. Right, TBHs summed over the first (half) 50 DNMS trials of CX516 session day 25 for comparison with day 8. Note enhancement of Pre-CX516 pattern, but neurons continued to fire in single-event mode. However, composite TBH (bottom) exhibited nearly the same overall ensemble firing pattern as the composite TBH for the carryover group (Fig. 2). Inset, Waveforms show the discriminated extracellular action potentials for each neuron on the respective recording days. Calibration: $50 \mu \mathrm{V}, 200 \mu \mathrm{sec}$. 

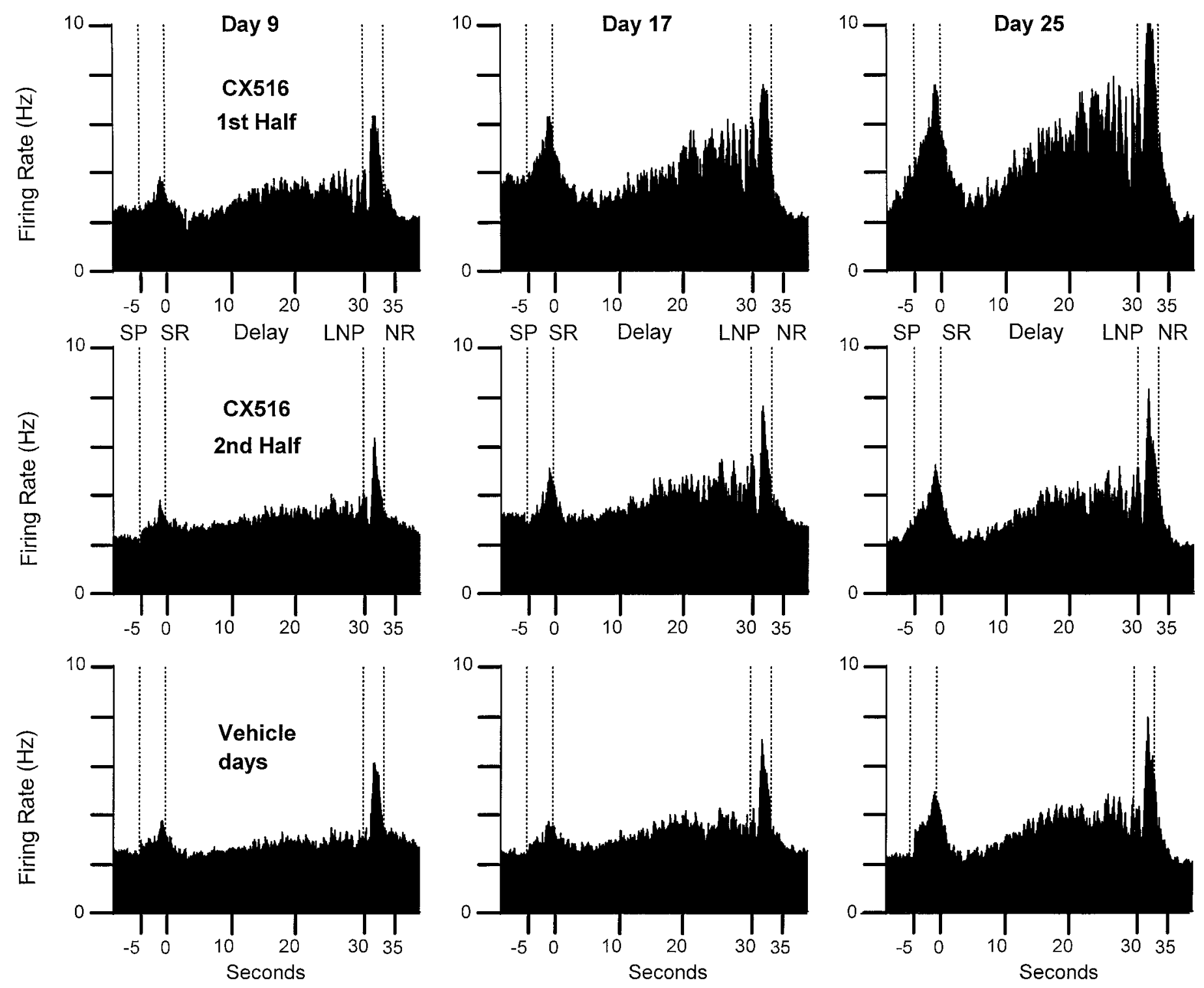

Figure 6. Composite TBHs of ensemble firing patterns from another animal in the noncarryover group constructed from eight hippocampal neurons in an ensemble with heterogeneous firing patterns (see Fig. 5). Composite TBHs are separated into first and second half drug session firing (50 trials each) and the next intervening vehicle session (first 50 trials), on days 9 (first drug day), 17 (fifth drug day), and 25 (last drug day). Note marked reduction in firing during second half and vehicle sessions as well as reduction in carryover to vehicle session. Composite TBHs averaged across noncarryover ensembles were constructed the same as in Figures 2 and 5.

the same drug session, and on the following vehicle day, firing in the ensemble was considerably decreased. Interestingly, the ensemble firing in the second half of the session of day 25 near the end of drug treatment and on the following vehicle day (day 26) closely resembled firing in the first half of the session during early exposure to the drug (day 9, top), whereas firing in the first half of the session (day 25, top), was markedly elevated in all phases of the task. Thus, firing was progressively increased across ensembles of neurons (Fig. 5) (even on nondrug days) in the noncarryover group by CX516, but the changes were not associated with alterations in single event firing patterns, as in the carryover group.

\section{Selective effects of CX516 on delay firing: correct versus error trials}

As described above (Fig. 1D), overall firing rate in the Delay was markedly and selectively increased on long versus short delay trials. The necessity to understand the significance of Delay firing for performance required sorting Delay firing by Correct versus Error trials. In the Pre-CX516 sessions, overall firing rates within the Delay did not differ for Correct or Error trials (Pre-CX516 correct trials $=1.91 \pm 0.43 \mathrm{~Hz}$; Pre-CX516 error trials $=2.08 \pm$ $0.51 \mathrm{~Hz} ; F_{(1,800)}=0.89$; NS). However, after CX516 administration there was a marked difference (Post-CX516 correct trials = $5.88 \pm 0.72 \mathrm{~Hz}$; Post-CX516 error trials $=2.37 \pm 0.61 \mathrm{~Hz} ; F_{(1,800)}$ $=92.81 ; p<0.001)$. Furthermore, an analysis of covariance (ANCOVA) revealed that firing in the Delay interval $(>20 \mathrm{sec})$ after CX516 exposure became correlated on a day-to-day basis with successful DNMS performance $\left(F_{(1,286)}=5.60 ; p<0.01\right.$; ANCOVA). As shown in Figure $1 B$, this correlation followed the emergence of increased firing in the Sample phase on the same days that DNMS performance began to improve $\left(F_{(1,286)}=6.65\right.$; $p<0.01$; ANCOVA). Thus, increased Sample firing and behav- 

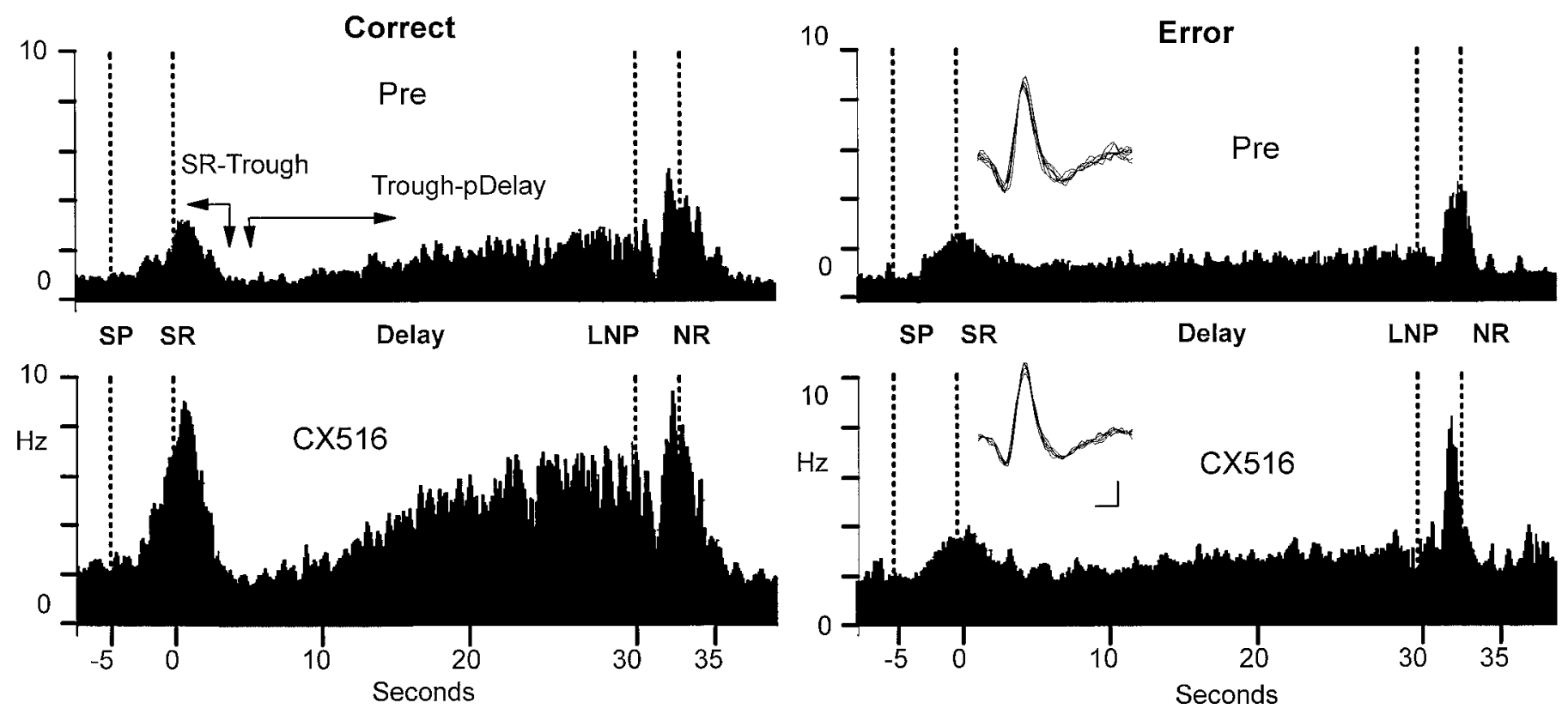

Figure 7. Distinction between single neuron TBH firing patterns on Correct versus Error trials. Pre, TBHs from a single neuron recorded from an animal in the CX516 carryover group reconstructed from long delay (30-40 sec) correct (left) or error (right) trials on day 8 of testing (Pre-CX516). Note the reduced SR and ramp-like firing in the Delay but maintained NR firing on error trials. $C X 516$, TBHs from same neuron show enhanced differences in firing between correct and error trials recorded on day 25 of testing (CX516). Note similarity in error trial TBHs before and during CX516 administration. Inset shows extracellular action potential waveforms used to construct TBHs recorded from the same electrode on days 8 (Pre) and 25 (CX516). Calibration: $50 \mu \mathrm{V}, 200 \mu \mathrm{sec}$. Arrows indicate differential firing rate measures used to determine changes in firing topography across the delay interval. SR-Trough, Difference between peak firing during the Sample phase, and minimum firing "trough" in the early (5-10 sec) portion of the Delay. Trough-pDelay, Difference between the same trough measure and the peak (maximal) firing achieved afterward within the delay (20-40 sec to LNP).

ioral improvement preceded slightly the alterations in Delay firing associated with the drug. This would be expected, given the demands of the DNMS task, if firing in the delay is representative of what is encoded in the Sample phase (Deadwyler et al., 1996a).

The above correlation suggests that firing across the Delay within a trial differentiated Correct versus Error trials. These differences were detected and are illustrated in Figure 7 as changes in the topography of firing during the Delay interval. Two measures captured the important topographical features of Delay firing on Correct versus Error trials. The first was the subsequent difference in firing between the peak of the SR and the rapid decline or "trough" that occurred between 0 and $10 \mathrm{sec}$ after the SR peak (Fig. 7, SR-Trough measure). The second measure was the difference in firing from the Trough to subsequent peak firing in the Delay interval measured at the LNP (Fig. 7, Trough-pDelay). Both measures revealed highly significant differences in firing topography in the Delay between Correct and Error trials in the Pre-CX516 condition (SR-Trough: Pre-CX516 Correct $=1.64 \pm 0.17 \mathrm{~Hz}$ vs Pre-CX516 Error $=0.57 \pm 0.15 \mathrm{~Hz}$; $F_{(1,800)}=6.17 ; p<0.01$; Trough-pDelay: Pre-CX516 Correct $=$ $0.82 \pm 0.11 \mathrm{~Hz}$ vs Pre-CX516 Error $=0.12 \pm 0.06 \mathrm{~Hz} ; F_{(1,800)}=$ $7.17 ; p<0.001)$. The SR-Trough difference was $66 \%$ smaller on Error than on Correct trials, whereas the Trough-pDelay measure was reduced by $85 \%$ on Error versus Correct trials. These differences are shown for the carryover group in Figure $8 A$. There were no differences in any of these measures between Pre-CX516 carryover and Control group Delay firing.

After CX516 administration, major increases appeared in both of these measures for Correct trials only. For the SR-Trough, CX516 Correct trial mean increased to $482 \%$, and for TroughpDelay the increase was $643 \%$ relative to the Pre-CX516 Correct values $\left(F_{(1,800)}>171.03\right.$ (both); $\left.p<0.001\right)$. These same two measures for Error trials showed only minimal (SR-Trough CX516 Error increases $\left.102 \% ; F_{(1,800)}=6.41 ; p<0.01\right)$ or no changes (Trough-pDelay CX516 Error was not significant) during CX516 administration. On day 17 (fifth day of CX516 administration) there was a marked increase in the Trough-pDelay measure and corresponding smaller daily increments in the SRTrough measure over the remainder of CX516 sessions (Fig. 8A). These same differences existed on intervening drug days and in the Post-CX516 period. Figure $8 A$ also shows that DNMS performance (dotted line) closely tracked the increases in Delay firing on Correct trials as represented by the two measures. Finally, the same two measures failed to indicate any comparable changes (with the exception of the small increase in T-pD on day 17) in Delay firing on Error trials over the same time period.

Figure $8 B$ shows the same two measures for the noncarryover group taken over the first half of the session only, when the drug effects were most prominent. It is clear that neither the SRTrough $(S R-T)$ nor the Trough-pDelay $(T-p D)$ measures were elevated to the same degree over the time course of CX516 administration as in the carryover group (Fig. $8 A$ ). In addition, the oscillatory nature of the changes on drug versus vehicle days shows that delay firing on Correct trials was also susceptible to the lack of persistence of drug effects in the same manner as reflected in the behavioral performance of this group (dotted line). Vehicle day firing is shown as solid symbols, reflecting a significant increase in Correct trial Delay firing over Pre-CX516 levels, but not to the same extent as in the first half of the session. As in the carryover group, changes in firing on Error trials were also minimal across the entire $32 \mathrm{~d}$ CX516 testing period.

The marked increases in Delay firing shown in Figure $8 A, B$ were not present in the Control group at any stage of the study, which is consistent with the corresponding lack of behavioral 

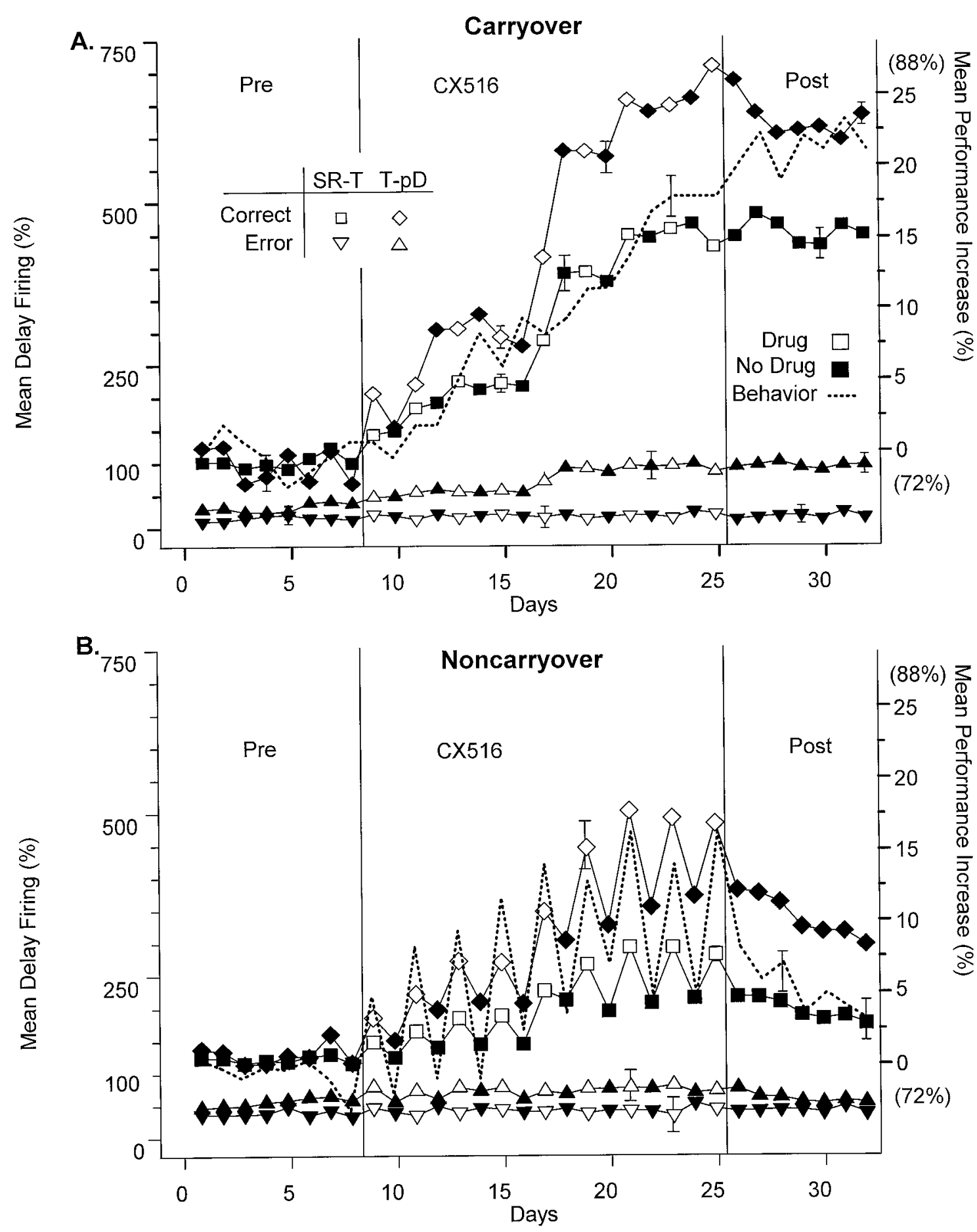

Figure 8. Mean firing rate changes in the Delay interval between Correct and Error trials over all hippocampal neurons recorded from animals in the carryover versus noncarryover groups. A, Carryover, SR-Trough $(S R-T)$ and Trough-pDelay $(T-p D)$ measures of Delay firing (see Fig. 7) were assessed on 30-40 sec delay trials for the Carryover group. Symbols indicate the mean rate ( \pm SEMs) of increase across animals for correct (square and diamond) and error trials (regular and inverted triangles), as a percentage of predrug firing on correct trials. Because most error trials did not show distinct "troughs" (Fig. 7), SR-T and T-pD means were below correct trial levels. Open symbols (Drug) indicate CX516 administration days; filled symbols (No Drug) indicate vehicle-only days. The dashed line indicates mean percent increase in correct DNMS behavioral performance (right axis) on the same trials for all nine animals. Error bars indicate largest SEMs for each curve. B, Noncarryover, SR-T and T-pD measures of Delay firing for the first 50 trials each day for the noncarryover group. Axes are scaled the same as in $A$ to illustrate differences in mean firing across all 20 neurons and DNMS performance increase over $32 \mathrm{~d}$ testing period. Only the first 50 trials per session were used to calculate CX516 firing rate and DNMS performance measures. Firing rate and performance during the second 50 trials on drug days (not shown) were reduced to similar levels as on nondrug days (filled symbols). After cessation of CX516 treatment, both SR-T and T-pD measures were elevated but decreased steadily toward baseline. Symbols and axes are the same as in $A$. 

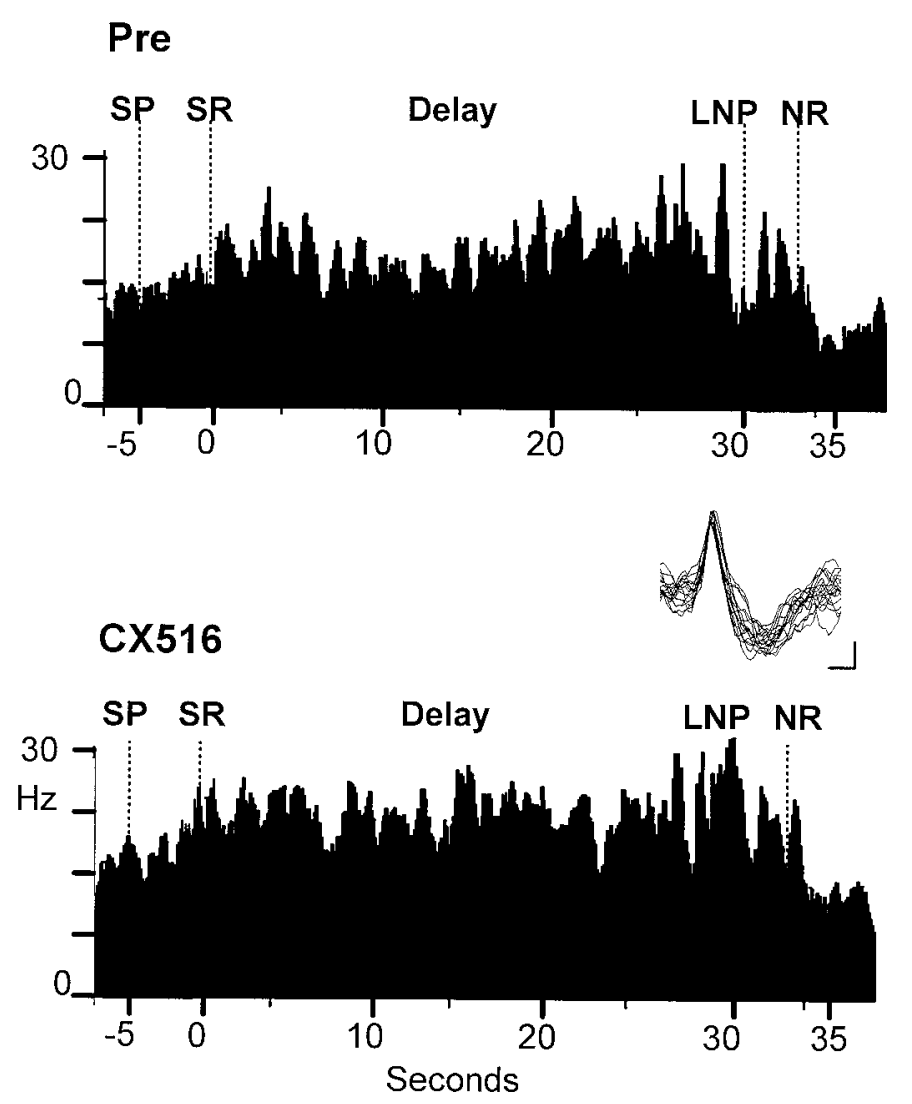

Figure 9. Firing changes of hippocampal theta cell during DNMS trials before and after CX516 administration. Top, TBH for a CA1 hippocampal theta cell averaged over all 100 DNMS trials on day 8 (Pre-CX516). Bottom, TBH for the same theta cell on day 25, after CX516 administration. Inset shows the extracellular action potential waveform. Calibration: $50 \mu \mathrm{V}, 200 \mu \mathrm{sec}$.

improvement over the same time course (Fig. $1 A$ ). The specificity of CX516 action on relevant neural processes was revealed by examination of the firing of simultaneously recorded theta cells (Fox and Ranck, 1981) before and after drug administration in these same animals (Fig. 9). Although the overall firing rate for the theta cell shown in Figure 9 was increased from $14.8 \pm 2.1$ to $20.3 \pm 2.3 \mathrm{~Hz}$, there was no specific change in firing during particular DNMS task-relevant events. This was true for six theta cells recorded across five different animals.

\section{Effect of CX516 on firing correlates of proactive interference}

Previous studies have demonstrated that after long delay trials in which an error is committed [long delay error (LDE) trials], there is a strong tendency for animals in this task to change their behavioral response strategy which "proactively interferes" with performance on the next trial (Hampson and Deadwyler, 1996a; Hampson et al., 1998). Such a shift in strategy reflects an attempt to maximize the (chance) probability that the next trial presented after the LDE will provide a strongly encoded SR that can survive the potential occurrence of a second long delay trial. To accomplish this, animals deliberately encode the next Sample response (regardless of position) the same as the position of the error on the last (LDE) trial. The strategy generates a performance curve after an erroneous lever response (NR in the LDE) that is nearly identical to the probability of presentation of the "same" or "different" lever (SP) in the Sample phase of the next trial (Hampson and Deadwyler, 1996a; Hampson et al., 1998). As shown in the companion article (Hampson et al., 1998), the tendency to invoke this strategy was virtually abolished after CX516 administration (Hampson et al., 1998, their Fig. 6). It therefore becomes important to determine whether the reduction of this tendency was accompanied by an alteration of hippocampal neuron activity that could be related to CX516's abolishment of this proactive interference effect.

Because the marked shift in strategy occurred after an LDE trial, we examined firing in the ITI between the end of an LDE and the Sample phase of the next trial, where the strategy shift was invoked. In the absence of drug there was a significant elevation in ITI firing after LDE trials compared with Correct trials (PreCX516 LDE ITI $=3.34 \pm 0.16 \mathrm{~Hz}$; Pre-CX516 Correct ITI = $\left.1.23 \pm 0.16 \mathrm{~Hz} ; F_{(1,800)}=28.17 ; p<0.001\right)$. This resulted in distinct peak-to-trough and overall ITI firing rate differences after the NR on Correct versus Error trials.

More important, however, were the transitions in firing between the ITI and SR on the next trial. After firing was reduced in the ITI after the NR on a previous Correct trial, it then "ramped up" to differential peak latencies, from the SP, depending on the position of the lever responded to in the Sample phase (Fig. 4). Average Sample peak discharge latencies on Correct trials for six of nine ensembles recorded from carryover animals for right $(3.93 \pm 0.27 \mathrm{sec})$ versus left $(6.71 \pm 0.54 \mathrm{sec}) \mathrm{SR}$ positions were significantly different from each other $\left(F_{(1,231)}=\right.$ 14.53; $p<0.001)$. The other three ensembles encoded right and left lever positions on Correct trials with peak latencies that were the opposite of those. Therefore all animals exhibited shifts in peak latency as a means of encoding the position of the SR on Correct trials (Fig. 10). On the trial after an LDE, the peak latencies were significantly reversed $\left(F_{(1,231)}=11.33 ; p<0.001\right)$ and did not correspond to the actual position of the lever in the Sample phase $\left(F_{(1,231)}=0.15 ; p=0.67\right)$. Thus, the SR of the next trial after an LDE was "miscoded" in terms of the wrong latencyto-peak firing. This is shown in the split-half TBHs in Figure 10 as a lack of correspondence between the location of the triangle and asterisk and the firing peak latencies associated with the SRs of the next trial (Fig. 10, Pre, top and bottom). This miscode was the same position as the NR (error) on the previous LDE (Fig. $10, N R$, top and bottom), occurred on $\sim 50 \%$ of trials, and correlated behaviorally with the shift to a maximization strategy (Hampson et al., 1998). In accordance with the strategy, if the Sample lever was the same position as the NR (error) in the previous LDE trial, that SR was encoded correctly. Thus, miscodes that occurred in the Sample phase of the next trial resulted from a mismatch between the position of the NR (error) in the preceding LDE and the current SR and was likely the basis for the proactive interference effect (cf. CX516, Fig. 10, top and bottom).

As shown in the companion article (Hampson et al., 1998), CX516 markedly reduced proactive interference and the tendency to invoke this maximizing strategy. A change in firing after LDE trials would be consistent with the above hippocampal cell correlates of interference with sample encoding. After exposure to CX516, the firing pattern after LDE trials was virtually identical to that obtained on Correct trials (CX516 mean LDE = $2.47 \pm 0.24 \mathrm{~Hz}$; CX516 mean Correct $=2.58 \pm 0.23 \mathrm{~Hz} ; F_{(1,800)}$ $=0.07 ; p=0.79)$. In addition, after CX516 exposure there were no significant "miscodes" of SR peak latencies on trials after LDE trials $\left(F_{(1,231)}=0.11 ; p=0.74\right)$, as shown by their alignments with the asterisk and triangle in Figure 10 (CX516). CX516 blockade of these proactive changes in firing tendency in hippocampal 

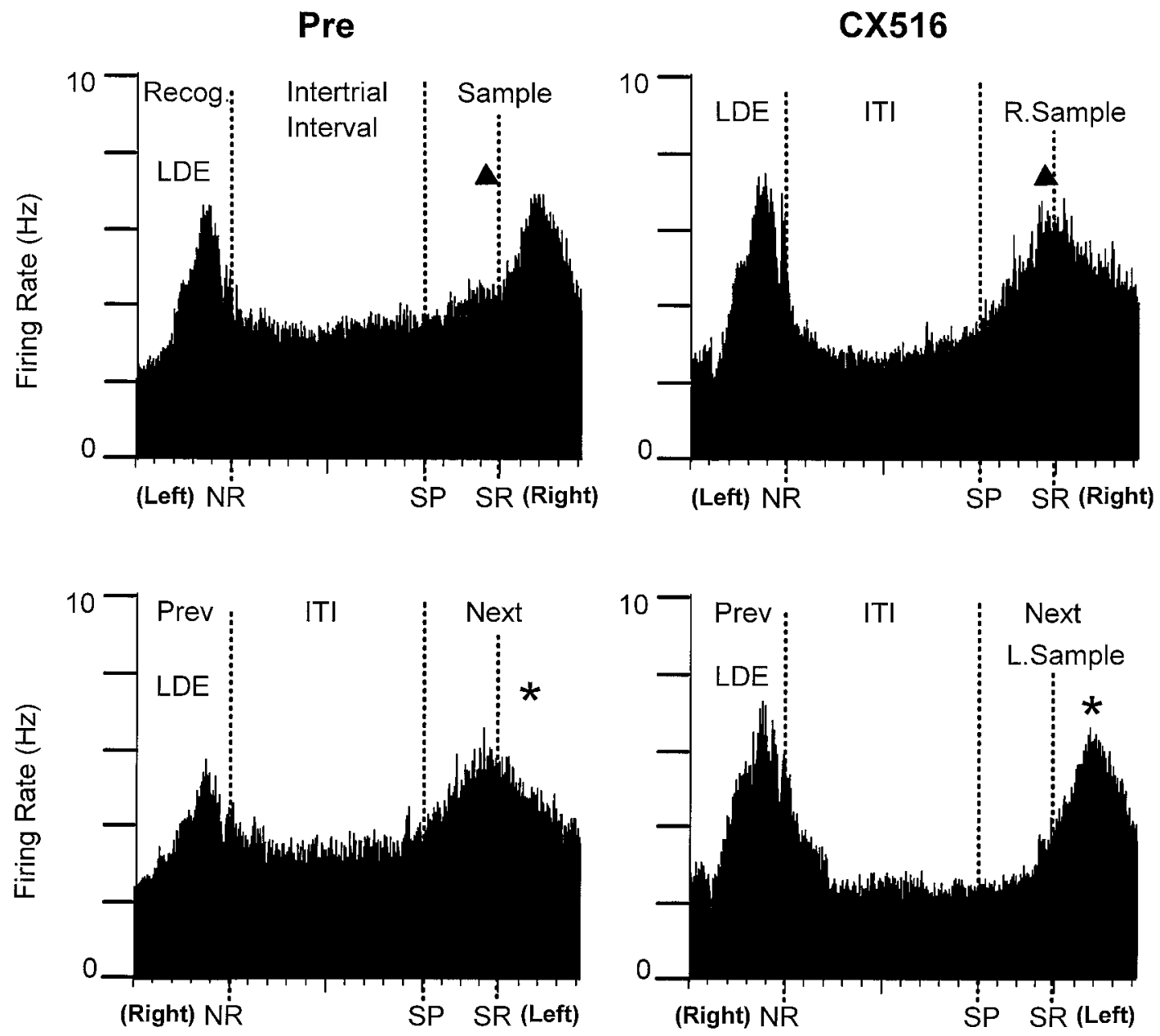

Figure 10. Proactive influence of LDEs on task-relevant hippocampal cell firing. Split-half composite TBHs show continuous neural activity from the last part (Recognition Recog phase) of a previous (Prev) LDE trial, across the intervening Intertrial Interval (ITI), and then terminating after the Sample phase of the subsequent (Next) trial. Pre, The split-half TBHs on the left are from day 8 (Pre-CX516) of testing and show firing peaks at the NR (error) responses (top $\mathrm{TBH}=$ Left; bottom $\mathrm{TBH}=$ Right) at the termination of the (Prev) LDE trial and the corresponding latency differentiated firing peaks to the SR $($ top $=$ Right; bottom $=$ Left $)$ in the Sample phase on the subsequent (Next) trial. Locations of symbols are associated with latency of peak firing on Correct trials: triangle, SR (Right); asterisk, SR (Left). CX516, Split-half TBHs on day 25 of testing (last day of CX516 administration). Similar Left (top) and Right (bottom) NRs (errors) and firing are shown on the preceding LDE trial. Peak firing patterns for the Right (top) and Left (bottom) respective SRs on the subsequent (Next) trial are labeled as to appropriate encoding of position (top $=$ R.Sample; bottom $=$ L.Sample) determined by the fact that the same peak firing patterns occur on correct trials. The time course of each split-half TBH was synchronized at the nonmatch response (NR) and averaged over six hippocampal ensembles $(n=37$ neurons) from six different animals in the carryover group. The intertrial interval (ITI) between $\mathrm{NR}$ and presentation of the sample lever $(S P)$ was $10 \mathrm{sec}$ for each trial (tick marks $=1 \mathrm{sec}$ ). The mean duration of the interval from SP to sample response $(S R)$ was $4.1 \pm 0.27 \mathrm{sec}$ for all trials and hence did not vary for Left versus Right SRs. LDE, Long delay error trial; SP, sample lever presentation; ITI, intertrial interval; $S R$, sample lever response; $N R$, nonmatch response; Recog., recognition phase; Sample, sample phase; Prev, previous trial; Next, trial after the LDE and ITI; triangle, correct peak latency for Right SR; asterisks, correct peak latency for Left SR.

neurons is therefore a candidate mechanism for the observed disappearance of the behavioral strategy shift after LDE trials reported in the companion article (Hampson et al., 1998).

\section{DISCUSSION}

\section{Enhanced hippocampal activity and DNMS performance}

Previous studies have shown the necessity of an intact hippocampus to perform this DNMS task (Dunnett, 1989). More recently it was shown that ibotenate lesions that remove the entire hippocampus and spare retrohippocampal structures seriously impair DNMS behavior in this task in a delay-dependent manner (Hampson et al., 1995). The issue examined by these recording studies is therefore not whether the hippocampus contributes to DNMS performance but rather, which aspects of hippocampal activity are critical to that performance. The ampakine CX516 caused unprecedented improvement in DNMS performance and did so with considerable behavioral selectivity (Hampson et al., 1998). Drugs such as CX516 therefore have obvious use in determining the connection between hippocampal neural activity and performance.

The electrophysiological analyses described here collectively indicate that several definitive changes in hippocampal cell firing occurred after the alternating day treatment regimen with CX516. Those changes were positively correlated and consistent with improved DNMS performance in the same animals (Hampson et al., 1998). In no instances were the hippocampal firing correlates described above seriously dissociated from the behavioral effects of the drug. Further evidence for a functional relationship between changes in hippocampal cell activity and im- 
proved DNMS performance came from the fact that both the behavioral and physiological effects of CX516 showed similar time courses over treatment days (Fig. 1). Finally, the ampakine enhanced a pattern of activity associated with correct (but not error) performance in the task that was differentially expressed on more difficult to perform trials with long delays.

\section{CX516-induced firing changes in hippocampal cells}

Examination of the effects of CX516 on hippocampal cell firing helps to explain how the ampakine facilitated DNMS performance. The progressive daily facilitation of behavior and the concomitant enhancement of hippocampal neuron activity suggest that the ampakine CX516 targeted hippocampal glutamatergic processes involved in successful DNMS performance. As shown in Figure 1, enhanced cell firing appeared differentially in the three task phases (first in the Sample phase, 1-2 d later in the Delay phase, and finally $2-4 \mathrm{~d}$ later in the Recognition phase). This succession of changes is consistent with the order of taskrelevant events within a trial and suggests that enhanced firing in earlier phases (Sample and Delay) was necessary to provoke changes in the later phase (Recognition). It therefore may be necessary for performance to be facilitated before firing is incremented in these later phases of the task. The significance of these progressive changes may lie in the interaction between alterations in performance that accompany Sample firing and potential feedback to alter firing in the Delay and Recognition phases.

The increase in the proportion of multi-event neurons from 29 to $78 \%\left(\chi^{2}=14.7 ; p<0.01\right)$ in the carryover group indicates that CX516 permanently altered the mode of hippocampal cell firing to include additional critical task relevant events (Fig. 4). We have defined such firing tendencies within hippocampal neurons as "disjunctive" (Eichenbaum et al., 1994; Hampson and Deadwyler, 1996b), which under normal circumstances exists in a small percentage $(<30 \%)$ of recorded hippocampal neurons (Hampson et al., 1993). Multi-event (disjunctive) neurons encode at least two task-relevant events differentially on any given trial, whereas single-event neurons encode only one. The net effect of this change in proportion of multi-event neurons would be to increase the "redundancy" of the information encoded across the same ensemble of cells on any given trial (Figs. 2, 5). Perhaps more importantly, the increased proportion of multi-event neurons as seen in carryover animals (Fig. 2) would allow for effective pattern encoding by a smaller number of (disjunctive) neurons in the ensemble than ensembles of single-event neurons. Allocation of such additional encoding tendencies to neuron ensembles in carryover animals could well improve DNMS performance, because as we have documented previously, "strong" (i.e., redundant) Sample "codes" within hippocampal populations are more likely to persist across long delay intervals than weak codes (Hampson and Deadwyler, 1996a).

\section{Pharmacologically dependent effects of CX516}

For unknown reasons, in 3 of the 12 animals tested, CX516 influenced behavior and hippocampal activity over a time period consistent with the known metabolic half-life of the ampakine in the brain (Hampson et al., 1998), roughly 35-40 min of the testing session. Although it is obvious that the same facilitative effects of the drug were present (but truncated) in the noncarryover group, it is not at all clear why both performance and associated cell firing rates appeared to depend closely on the pharmacokinetics of drug action in these and not other animals (Fig. 6). It is possible that the same basic mechanism(s) was triggered in both groups in the presence of the drugs (Staubli et al., 1994a; Rogers, 1997), but for some reason were not maintained or converted to permanent status in the noncarryover animals. With respect to hippocampal activity, some insight is gained from the fact that only $25 \%$ of hippocampal neurons (in contrast to $78 \%$ in the carryover group) were converted to multi-event (disjunctive) firing status in the noncarryover group even during behaviorally facilitated portions of the drug session (Fig. 5). The enhanced DNMS performance was attributable largely to the elevated firing of single-event cells (Fig. 5) that did not persist beyond the direct pharmacological actions of the drug (35-40 $\mathrm{min}$ ) in the noncarryover group (Fig. 6). Such drug-induced firing may have been sufficient to generate the necessary task-relevant firing patterns across the entire ensemble (Fig. 5), but only while drug levels remained high. Because multiple task-relevant features were not encoded in single neurons, increased levels of performance in noncarryover animals may not have been possible without the ensemble synchronization provided by the ampakine.

\section{Effects of CX516 on hippocampal activity related to reduced proactive interference}

The proactive influence generated in trials (of any length delay) that immediately followed LDE trials was substantially reduced by CX516. Before CX516, the differentiated temporal firing patterns miscoded the lever pressed in the Sample phase of the next trial (Fig. 10) (Hampson and Deadwyler, 1996a). This generated errors on the trials that were different from the preceding LDE and correct responses that were the same, regardless of delay (Hampson et al., 1998, their Fig. 6). After CX516, clearly differentiated temporal firing patterns were correctly generated to either left or right lever position responses (SR) in the Sample phase after LDE trials (Fig. 10, CX516). Indeed, the reduction in the number of miscode errors on trials after LDEs and the resultant elimination of proactive interference in DNMS performance were some of the more dramatic effects of exposure to the ampakine. The fact that CX516 specifically targeted the "miscode" effect after LDEs provided insight into a neurophysiological process that interfered directly with encoding, and constituted the basis for the observed behavioral expression of proactive interference.

\section{Effects of ampakines on neuronal processes}

Studies of excised patches from neuronal membranes have shown that positive modulators of AMPA/KA-type glutamate receptors produce a mixture of two effects: (1) reduction in the rate at which the receptors desensitize and (2) a slowing of the deactivation time leading to prolongation of currents elicited by very brief $(1 \mathrm{msec})$ pulses of AMPA receptor agonists (Arai et al., 1995). Cyclothiazide and other benzothiadiazides markedly affect desensitization of receptor-gated processes but not deactivation of AMPA receptors. Ampakines, however, alter both processes with proportional affects varying between the two types of drugs (Arai et al., 1995; Partin et al., 1995, 1996). Similar analyses indicate that changes in the deactivation rate are attributable to a change in channel opening-closing rates or a reduction in the transmitter dissociation rate constant for agonist binding to the receptor or both (Partin et al., 1996). CX516 is unusual among the ampakines because the balance of its actions are shifted toward slowing deactivation (Arai et al., 1995).

Given the above action of ampakines in modifying AMPA channel properties, repeated exposure may have produced longterm functional changes in hippocampal cell firing in the DNMS 
task. The effects of ampakines such as CX516 on desensitizationdeactivation of AMPA channels in hippocampal slices shift the dose-effect curve for glutamate to the left, making smaller quantities of glutamate more effective in producing ligand-gated extracellular current (Lynch and Baudry, 1991; Larson et al., 1995). It is noteworthy, therefore, that reported concentrations of ampakines as well as the relative potencies of different ampakines on AMPA receptor-mediated current in patch-slice studies are predictive of the dosages and blood levels needed to elicit behavioral changes (Staubli et al., 1994a,b; Rogan et al., 1997; Hampson et al., 1998). Because ampakines such as CX516 readily cross the blood-brain barrier (Rogers, 1997), AMPA receptors with fast excitatory transmission are the probable sites involved in the effects described here.

\section{Potential mechanisms of ampakine facilitation of DNMS performance}

Any of the above ampakine-sensitive processes could be either directly or indirectly responsible for the observed changes in DNMS task-relevant firing of hippocampal neurons. The relatively "weak" differentiation between Correct and Error trials under nondrug conditions shown in Figures 5, 6, and 8 (PreCX516) suggests that the majority of hippocampal neurons do not normally participate fully in encoding information in DNMStype tasks. Substantial evidence exists for Sample encoding in short-term memory tasks in other anatomically related structures, such as perirhinal cortex or parasubiculum (cf. Eichenbaum et al., 1994); therefore hippocampal participation may be required to some extent but is not normally sufficient to maintain performance. However, after exposure to the ampakine, encoding by hippocampal neurons was altered drastically to represent more than one event within a trial and to fire in more than one phase of the task (Fig. 4). This could have reduced the "difficulty" of the task in animals given CX516 via improved delay-dependent representation of stimulus properties in task-activated hippocampal cells (Hampson et al., 1998, their Fig. 5).

The building of new and the strengthening of existing associations by the ampakines, in addition to explaining improved DNMS performance, might also account for the persistence of the improved performance in the absence of ampakine. That is, the consequences of enhanced "learning" that were readily expressed (on nondrug days) without further contribution from the ampakine may have resulted from associations formed in previous (drug) testing sessions. Mechanistically, ampakines through their known fast glutamatergic excitatory action could facilitate hippocampal cell firing to other task-relevant cues, associations, or representation (Staubli et al., 1994b), effectively changing such cells from single- to multi-event status (Fig. 4). Calculations indicate that only a small percentage of the cells in a sparsely connected neural network, like hippocampus, will receive a sufficient number of inputs from any given "sensory event" necessary for their activation (Lynch and Granger, 1991; Granger et al., 1994, 1996). Thus the majority of "neurons" recorded would be expected on this basis to show single event correlates and be sparsely distributed within the ensemble (much like the PreCX516 ensemble firing shown in Figs. 2 and 5).

Drugs that positively modulate AMPA receptors increase the degree of depolarization at each active synapse, a condition that could lower the number of inputs required for hippocampal cell activation (Arai and Lynch, 1992). Applied to the current DNMS context, ampakines, by facilitating fast excitatory transmission (Arai et al., 1995), could amplify weak sensory inputs on single- event cells into an effective range to convert them to multi-event firing status through known associative mechanisms. The recent studies of polysynaptic potentiation by Yeckel and Berger (1998) demonstrate that the ampakine need only affect one set of synapses within a circuit to promote potentiation in other synaptic connections because this would be sufficient to alter the temporal patterning of inputs to "downstream" cells. This notion is supported by the findings here showing that changes in Sample phase firing appeared to be "permissive" for subsequent changes in activity in the Delay and Recognition phases of the task (Fig. 1B). Modeling studies (Lynch and Granger, 1991) have shown that "relaxing" the input requirements to hippocampal neural networks has the effect of broadening the range of signals or inputs to which cells are able to respond. Thus, the positive modulatory effects of ampakines might allow circumvention of existing limitations on hippocampal cell responsiveness that under nondrug conditions cannot be violated because of "biological constraints" imposed by the fixed density of connections within hippocampus.

In summary, performance of the DNMS task after exposure to CX516 was facilitated to a large degree by providing immunity to three major sources of errors that normally suppress responding in the DNMS task. First, CX516 maximized encoding of the Sample response. Second, it maintained representation of the Sample response at an amplified level across critical (i.e., later) portions of the intervening Delay interval. Third, it prevented proactive interference from selective types of previous error trials. These changes are consistent with the hypothesis that hippocampal neuronal activity participates critically to encode and represent events in the DNMS task. These findings, together with the initial encouraging evidence that CX516 is also effective in humans (Lynch et al., 1996), provide an expectation that many of the deficiencies resulting from loss of short-term memory might be reversible by ampakines such as CX516.

\section{REFERENCES}

Arai A, Lynch G (1992) Factors regulating the magnitude of long-term potentiation induced by theta pattern stimulation. Brain Res 598:173-184.

Arai A, Silberg J, Kessler M, Lynch G (1995) Effect of thiocyanate on AMPA receptor mediated responses in excised patches and hippocampal slices. Neuroscience 66:815-827.

Cahusac PMB, Miyashita Y, Rolls ET (1989) Responses of hippocampal formation neurons in the monkey related to delayed spatial response and object-place memory tasks. Behav Brain Res 33:229-240.

Cahusac PMB, Rolls ET, Miyashita Y, Niki H (1993) Modification of the responses of hippocampal neurons in the monkey during learning of a conditional spatial response task. Hippocampus 3:29-42.

Colombo M, Gross C (1994) Responses of inferotemporal cortex and hippocampal neurons during delayed matching to sample in monkeys (Macaca fascicularis). Behav Neurosci 108:443-455.

Deadwyler SA, Hampson RE (1995) Ensemble activity and behavior: what's the code? Science 270:1316-1318.

Deadwyler SA, Hampson RE (1997) The significance of neural ensemble codes during behavior and cognition. In: Annual review of neuroscience (Cowan WM, Shooter EM, Stevens CF, Thompson RF, eds). Palo Alto, CA: Annual Reviews.

Deadwyler SA, Bunn T, Hampson RE (1996a) Hippocampal ensemble activity during spatial delayed-nonmatch-to-sample performance in rats. J Neurosci 16:354-372.

Deadwyler SA, Byrd DR, Konstantopoulos JK, Evans GJO, Rogers G, Hampson RE (1996b) Enhancement of rat hippocampal ensemble activity by CX516 protects against errors in spatial DNMS. Soc Neurosci Abstr 22:1131.

Deadwyler SA, Rogers G, Lynch G, Hampson RE (1997) Facilitated encoding of task-relevant events in ensembles of hippocampal neurons by the ampakine CX516 (Cortex Pharmaceuticals). Soc Neurosci Abstr 23:509.

Dunnett SB (1989) Comparison of short-term memory deficits in animal 
models of aging using an operant delayed response task in rats. In: The biology of memory (Squire LR, Lindenlaub E, eds), pp 581-603. New York: FK Schattauer Verlag.

Eichenbaum H, Otto T, Cohen NJ (1994) Two functional components of the hippocampal memory system. Behav Brain Sci 17:449-518.

Fried I, MacDonald KA, Wilson CL (1997) Single neuron activity in human hippocampus and amygdala during recognition of faces and objects. Neuron 18:753-765.

Fox SE, Ranck Jr JB (1981) Electrophysiological characteristics of hippocampal complex-spike cells and theta cells. Exp Brain Res 41:399-410.

Fuster JM (1997) Network memory. Trends Neurosci 20:451-459.

Granger R, Whitson J, Larson J, Lynch G (1994) Non-hebbian properties of LTP enable high-capacity encoding of temporal sequences. Proc Natl Acad Sci USA 91:10104-10108.

Granger R, Deadwyler SA, Davis M, Moskovitz B, Kessler M, Rogers G, Lynch G (1996) Facilitation of glutamate receptors reverses an ageassociated memory impairment in rats. Synapse 22:332-337.

Hampson RE, Deadwyler SA (1996a) Ensemble codes involving hippocampal neurons are at risk during delayed performance tests. Proc Natl Acad Sci USA 93:13487-13493.

Hampson RE, Deadwyler SA (1996b) LTP and LTD and the encoding of memory in small ensembles of hippocampal neurons. In: Long-term potentiation, Vol 3, Ed 3 (Baudry M, Davis J, eds), pp 199-214. Cambridge, MA: MIT.

Hampson RE, Kirby MT, Alexander KE, King VC, Deadwyler SA (1992) Functional significance of anatomic connections between CA1 and CA3 hippocampal cells during delayed match to sample behavior in the rat. Soc Neurosci Abstr 18:1065.

Hampson RE, Heyser CJ, Deadwyler SA (1993) Hippocampal cell firing correlates of delayed-match-to-sample performance in the rat. Behav Neurosci 107:715-739.

Hampson RE, Byrd DR, Deadwyler SA (1994) Population analyses reveal that rat hippocampus encodes both spatial and task-specific information during delayed-nonmatch-to-sample tasks. Soc Neurosci Abstr 20:430.

Hampson RE, Byrd DR, Konstantopoulos JK, Bunn T, Jarrard LE, Deadwyler SA (1995) Proactive interference and short-term memory during performance of a DNMS task in normal rats and rats with hippocampus removed. Soc Neurosci Abstr 21:1215.

Hampson RE, Rogers G, Lynch G, Deadwyler SA (1998) Facilitative effects of the ampakine CX516 on short-term memory in rats: enhancement of delayed-nonmatch-to-sample performance. $\mathrm{J}$ Neurosci 18:2740-2747.

Heyser CJ, Hampson RE, Deadwyler SA (1993) The effects of delta-9$\mathrm{THC}$ on delayed match to sample performance in rats: alterations in short-term memory produced by changes in task specific firing of hippocampal neurons. J Pharmacol Exp Ther 264:294-307.

Larson J, Lieu T, Petchpradub V, LeDuc B, Ngo H, Rogers GA, Lynch G (1995) Facilitation of olfactory learning by a modulator of AMPA receptors. J Neurosci 15:8023-8030.

Lynch G, Baudry M (1991) Reevaluating the constraints on hypotheses regarding LTP expression. Hippocampus 1:9-14.

Lynch G, Granger R (1991) Serial steps in memory processing: possible clues from studies of plasticity in the olfactory-hippocampal circuit. In: olfaction as a model system for computational neuroscience (Eichenbaum H, Davis J, eds) Cambridge MA: MIT.
Lynch G, Kessler M, Rogers G, Ambros-Ingerson J, Granger R, Schehr RS (1996) Psychological effects of a drug that facilitates brain AMPA receptors. Int Clin Psychopharmacol 11:12-19.

McNaughton BL, O'Keefe J, Barnes CA (1983) The stereotrode: a new technique for simultaneous isolation of several single units in the central nervous system from multiple unit records. J Neurosci Methods 8:391-397.

Miller EK, Li L, Desimone RA (1991) Neural mechanism for working and recognition memory in inferior temporal cortex. Science 254:1377-1379.

Miller EK, Erickson CA, Desimone R (1996) Neural mechanisms of visual working memory in prefrontal cortex of the Macaque. J Neurosci 16:5154-5167.

Muller R (1996) A quarter of a century of place cells. Neuron 17:813-822.

Nishijo H, Ono T, Tamura R, Nakamura K (1993) Amygdala and hippocampal neuron responses related to recognition and memory in monkey. Prog Brain Res 95:339-357.

Otto T, Eichenbaum H (1992) Neuronal activity in the hippocampus during delayed non-match to sample performance in rats: evidence for hippocampal processing in recognition memory. Hippocampus 2:323-334.

Ono T, Nakamura K, Nishijo H, Eifuku S (1993) Monkey hippocampal neurons related to spatial and non-spatial functions. J Neurophysiol 70:1516-1529.

Partin K, Bowie D, Mayer ML (1995) Structural determinants of allosteric regulation in alternatively spliced AMPA receptors. Neuron 14:833-843.

Partin K, Fleck MW, Mayer ML (1996) AMPA receptor flip/flop mutants affecting deactivation, desensitization, and modulation by cyclothiazide, aniracetam, and thiocyanate. J Neurosci 16:6634-6647.

Rogan MT, Staubli UV, LeDoux JE (1997) AMPA receptor facilitation accelerates fear learning without altering the level of conditioned fear acquired. J Neurosci 17:5928-5935.

Rogers GA (1997) XIII International Symposium on Radiopharmaceutical Chemistry, Uppsala, June.

Rolls ET, Cahusac PM, Feigenbaum JD, Miyashita Y (1993) Responses of single neurons in the hippocampus of the macaque related to recognition memory. Exp Brain Res 93:299-306.

Sakurai Y (1990) Hippocampal cells have behavioral correlates during the performance of an auditory working memory task in the rat. Behav Neurosci 104:253-263.

Squire LR (1992) Memory and the hippocampus: a synthesis from findings with rats, monkeys, and humans. Psychol Rev 99:195-231.

Staubli U, Perez Y, Xu F-B, Rogers G, Ingvar M, Stone-Elander S, Lynch G (1994a) Centrally active modulators of glutamate receptors facilitate the induction of long-term potentiation in vivo. Proc Natl Acad Sci USA 91:11158-11162.

Staubli U, Rogers G, Lynch G (1994b) Facilitation of glutamate receptors enhances memory. Proc Natl Acad Sci USA 91:777-781.

Watanabe T, Niki H (1985) Hippocampal unit activity and delayed response in the monkey. Brain Res 325:241-254.

Yeckel MF, Berger TW (1998) Spatial distribution of potentiated synapses in hippocampus: dependence on cellular mechanisms and network properties. J Neurosci 18:438-450.

Zola-Morgan S, Squire LR (1993) Neuroanatomy of memory. Annu Rev Neurosci 16:547-563. 